\title{
Spin flips and precession in black-hole-binary mergers
}

\author{
Manuela Campanelli, ${ }^{1,2}$ Carlos O. Lousto, ${ }^{2,1}$ Yosef Zlochower, ${ }^{2,1}$ Badri Krishnan, ${ }^{3}$ and David Merritt ${ }^{1,4}$ \\ ${ }^{1}$ Center for Computational Relativity and Gravitation, School of Mathematical Sciences, Rochester Institute of Technology, \\ 78 Lomb Memorial Drive, Rochester, New York 14623, USA \\ ${ }^{2}$ Center for Gravitational Wave Astronomy, Department of Physics and Astronomy, The University of Texas at Brownsville, \\ Brownsville, Texas 78520, USA \\ ${ }^{3}$ Max-Planck-Institut für Gravitationsphysik, Albert-Einstein-Institut, Am Mühlenberg 1, D-14476 Golm, Germany \\ ${ }^{4}$ Department of Physics, 85 Lomb Memorial Drive, Rochester Institute of Technology, Rochester, New York 14623, USA
}

(Received 13 December 2006; revised manuscript received 29 January 2007; published 26 March 2007)

We use the "moving puncture" approach to perform fully nonlinear evolutions of spinning quasicircular black-hole binaries with individual spins unaligned with the orbital angular momentum. We evolve configurations with the individual spins (parallel and equal in magnitude) pointing in the orbital plane and $45^{\circ}$ above the orbital plane. We introduce a technique to measure the spin direction and track the precession of the spin during the merger, as well as measure the spin flip in the remnant horizon. The former configuration completes 1.75 orbits before merging, with the spin precessing by $98^{\circ}$ and the final remnant horizon spin flipped by $\sim 72^{\circ}$ with respect to the component spins. The latter configuration completes 2.25 orbits, with the spins precessing by $151^{\circ}$ and the final remnant horizon spin flipped $\sim 34^{\circ}$ with respect to the component spins. These simulations show for the first time how the spins are reoriented during the final stage of black-hole-binary mergers verifying the hypothesis of the spin-flip phenomenon. We also compute the track of the holes before merger and observe a precession of the orbital plane with frequency similar to the orbital frequency and amplitude increasing with time.

DOI: 10.1103/PhysRevD.75.064030

PACS numbers: 04.25.Dm, 04.25.Nx, 04.30.Db, 04.70.Bw

\section{INTRODUCTION}

There is widespread interest in understanding the dynamics of the last orbital stages of generic black-hole binaries (i.e. binaries with randomly aligned spins and unequal masses). These last few orbits before the binaries merge involve highly-nonlinear gravitational interactions leading not only to large amounts of gravitational radiation leaving the systems, but also to intricate coupling effects, particularly those involving the spins of the two component black holes. Questions of how the black holes orient their spins with respect to the (instantaneous) orbital plane and the extent to which the black holes spin-up during the last orbital stages are of great astrophysical interest. Spinning black holes are believed to be the engines of active galactic nuclei and quasars; the efficiency with which infalling matter is converted into radiation depends on the energy of the innermost stable orbit and is greatest for rapidlyspinning holes [1,2]. The energetic jets observed in many AGN and stellar-mass accreting systems are believed to be launched perpendicularly to the inner accretion disk, hence parallel to the spin axis of the accreting hole [3]. Changes in the spin direction are therefore potentially observable. A number of active galaxies exhibit semiperiodic deviations of the jet directions from a straight line [4], suggestive of precession of the accretion disk around the jet-emitting hole or geodetic precession of the larger hole, either of which might be driven by torques from a second orbiting black hole [5,6]. About 15 radio galaxies show jets with apparently abrupt changes in jet direction, forming $\mathrm{X}$ shaped patterns $[7,8]$. Jets of Seyfert galaxies often mis- align from subkiloparsec to kiloparsec scales [9]. Some models $[10,11]$ attribute these spatial variations to a sudden reorientation of the spin axis of the larger hole as it accretes a smaller hole. Fossil evidence that such reorientations were common in the past is observed in the nearly random orientations of jets in disk galaxies with respect to the disk plane $[12,13]$.

In the generic case, an inspiral of a black-hole binary should induce a precession in the direction of the spin axis of either hole [14], and unless the mass ratio is extreme, the final spin orientation is dominated by the orbital angular momentum of the binary, implying substantial reorientation as long as the spin and orbital angular momenta are initially misaligned [10]. We refer to this jump in the spin direction of the remnant, with respect to the individual spins, as a spin-flip. Note that this definition of spin-flip does not require nonlinear interactions; it is a simple consequence of the remnant acquiring most of the total angular momentum of the original binary. Also note that the "flip" is actually the difference in spins between two distinct types of objects: individual horizons and common horizons.

Aside from their astrophysical interest, black-hole binaries are one of the primary targets of the earth-based gravitational wave observatories, such as LIGO [15], VIRGO [16], GEO600 [17], etc. These detectors are operating at unprecedented sensitivities. In particular, the LIGO interferometers are currently taking data at their design sensitivities. The initial LIGO interferometers might be able to observe two $20 M_{\odot}$ inspiraling black holes out to 
a distance of more than $100 \mathrm{Mpc}$, and advanced LIGO could see the same event up to a cosmological redshift of $z \approx 0.4$ [18]. Predicted black-hole-binary gravitational waveforms will not only be of great help for the detection of this radiation using matched filtering techniques, but will also be essential for the interpretation of the signals, determination of the event rates, and extraction of astrophysical parameters. This will be especially important for the next generation of ground and space-based detectors, such as LISA $[19,20]$ which should observe gravitational wave bursts from the mergers of supermassive black holes in the centers of galaxies out to very high redshifts.

Simulations of the last orbital stages of black-hole binaries require solving the fully-nonlinear General Relativity field equations numerically on supercomputers. However, solving these nonlinear equations proved to be quite difficult and the problem remained unsolved for over 30 years. However, last year two independent techniques were developed that broke through the barrier of the numerical instabilities to produce spectacular results. In 2005 these two approaches were used to generate the gravitational waveforms from the last orbit of nonspinning, equalmass black-hole binaries. The first technique, which was developed by Pretorius [21], used a second-order formulation of the General Relativity field equations in a generalized harmonic gauge (GHG), along with singularity excision in the interior of the horizons, adaptive mesh refinement (AMR) on a compactified space, and the addition of constraint damping terms to the evolution equation. The second successful approach was developed a few months later by our group at UTB [22] and independently by the Numerical Relativity group at NASA/Goddard [23]. This latter method uses a mixed first-and-second-order formulation of the General Relativity field equations known as BSSN system [24-26], in combination with the puncture formalism [27,28] (without the need for singularity excision). This technique differs from previous work with punctures in that the punctures are not fixed on the grid. In both versions of this "moving puncture" approach modifications to the standard $1+\log$ lapse and Gamma-driver shift conditions were introduced [22,23,29].

Most of the groups in numerical relativity have now implemented one of the two approaches; the "moving puncture" technique being the more popular. Given its technical simplicity and flexibility, this latter approach has been used to produce several interesting results ranging from the original simulations of nonspinning equal-mass binaries [30-33] to spinning equal-mass binaries [34,35] and nonspinning unequal-mass binaries [36-38]. Notably, the moving puncture technique has recently also been successfully implemented in the case of neutron-starblack-hole-binaries [39,40], thus extending the impact of the technique on the numerical/astrophysical relativity community. Noteworthy, the GHG approach has also been successfully used to study eccentric orbits in blackhole binaries with equal-mass and small corotation spins [31]. A generalized harmonic form of Einstein's equations has also been successfully used together with a dual coordinates method to evolve black-hole-binary spacetimes for several orbits prior merger [41].

Alongside this impressive progress in computational and experimental relativity, the last few years have also seen significant mathematical progress in our understanding of black holes in full nonlinear general relativity. There is now a better understanding of the geometry and dynamics of trapped and marginally trapped surfaces using the quasilocal notions of trapping [42], isolated [43] and dynamical horizons [44]. Isolated horizons describe black holes in equilibrium in nonstationary spacetimes, and trapping and dynamical horizons describe the general time-dependent case. The applications of these ideas to classical and quantum black hole physics are too numerous to be described here, and we refer to [45-47] for reviews and a more complete set of references. For our present purposes, we are mostly interested in calculating the angular momentum of an (approximately) axisymmetric horizon. The calculation of angular momentum for isolated horizons is carried out using Hamiltonian methods as described in [48-50]. The analogous Hamiltonian calculation for nonstationary trapping and dynamical horizons is given in [51]. Conservation and balance laws describing how the horizon mass and angular momentum change in response to infalling matter/radiation are found in [52-55]. The calculation of the magnitude of black hole spin angular momentum tailored to numerical relativity is presented in [56] and more recently, [57] considers higher mass and angular momentum multipole moments. In this paper we use this formalism primarily to compute the direction and magnitude of the spin angular momentum vector.

This paper is organized as follows. In Sec. II we discuss the post-Newtonian predictions for spin and orbital plane precession. In Sec. III we describe the techniques used to evolve the binary and measure the horizon spins. In Sec. IV we describe the initial data parameters for the binary configurations mentioned in the remainder of the text. In Sec. V we give a detailed description of the new results regarding spin and orbit precession. In Sec. VI we discuss some of the implications of our results. Finally, in the appendix we review past results from aligned-spin and nonspinning binaries.

\section{POST-NEWTONIAN ANALYSIS}

If the spins of the two black holes in a binary are not aligned with the total angular momentum, then the spin and orbital angular momenta will precess about the total angular momentum. Precession of the spin of the holes is produced by spin-orbit coupling and the spin-spin coupling. This effect has been studied in several papers by means of the post-Newtonian expansion $[58,59]$ 


$$
\begin{aligned}
\frac{d \vec{S}_{i}}{d t}= & \frac{1}{r^{3}}\left\{\left(\vec{L}_{N} \times \vec{S}_{i}\right)\left(2+\frac{3}{2} \frac{m_{j}}{m_{i}}\right)\right. \\
& \left.-\vec{S}_{j} \times \vec{S}_{i}+3\left(\hat{n} \cdot \vec{S}_{j}\right) \hat{n} \times \vec{S}_{i}\right\},
\end{aligned}
$$

where $\vec{L}_{N} \equiv \mu(\vec{x} \times \vec{v})$ is the Newtonian orbital angular momentum, $\vec{x} \equiv \vec{x}_{1}-\vec{x}_{2}, r \equiv|\vec{x}|, \vec{v}=d \vec{x} / d t, \hat{n} \equiv \vec{x} / r$, $m=m_{1}+m_{2}, \quad \mu \equiv m_{1} m_{2} / m, \quad \eta \equiv \mu / m, \vec{S} \equiv \vec{S}_{1}+\vec{S}_{2}$, an over-dot denotes $d / d t$, and the $j$ subscript denotes the other hole. Since the time derivative of the individual spin of the holes has the form $\dot{\vec{S}}_{i}=\vec{\Omega}_{i} \times \vec{S}_{i}$ this implies that the magnitude of each individual spin is conserved and only its direction will continuously change in time and rotate at a precession frequency given by

$$
\vec{\Omega}_{i}=\frac{1}{r^{3}}\left\{\left(2+\frac{3}{2} \frac{m_{j}}{m_{i}}\right) \vec{L}_{N}-\vec{S}_{j}+3\left(\hat{n} \cdot \vec{S}_{j}\right) \hat{n}\right\} .
$$

Both the spin and orbital planes precess. This is due to the fact that, if the radiated angular momentum is neglected, the total angular momentum $\vec{J}=\vec{L}+\vec{S}$ is conserved. Hence the $\dot{\vec{L}}=-\dot{\vec{S}}$. Thus the orbital plane will precess at the same frequency as the total spin. The gravitational radiation reaction effect will generate a net loss of $J$ that will actually produce an increase in the amplitude of the spin and orbital oscillations since the black holes will get closer; magnifying the spin-orbit coupling. In this paper, we go beyond the post-Newtonian expansion and study these effects using full numerical evolutions.

Precession occurs in the plane perpendicular to the total angular momentum. We thus split the spin vector of each hole into components parallel to, and perpendicular to, the total angular momentum, i.e. $\vec{S}=\vec{S}_{\|}+\vec{S}_{\perp}$, where $\vec{S}_{\|}=$ $(\vec{S} \cdot \hat{J}) \hat{J}$ and $\vec{S}_{\perp}=\vec{S}-(\vec{S} \cdot \hat{J}) \hat{J}$. Note that the direction (but not magnitude) of the total angular momentum does not change significantly between the start and end of the simulations. We define the total angle of precession $\Theta_{p}$ as the angle by which $\vec{S}_{\perp}$ is rotated between the start and the end of the simulation. The total precession angle is then given by

$$
\cos \Theta_{p}=\frac{\vec{S}_{M} \cdot \vec{S}_{I}-\left(\hat{J} \cdot \vec{S}_{M}\right)\left(\hat{J} \cdot \vec{S}_{I}\right)}{\sqrt{\left[S_{M}^{2}-\left(\hat{J} \cdot \vec{S}_{M}\right)^{2}\right]\left[S_{I}^{2}-\left(\hat{J} \cdot \vec{S}_{I}\right)^{2}\right]}},
$$

where $\vec{S}_{I}$ is the initial spin of one of the individual horizons, $\vec{S}_{M}$ is the spin of that individual horizon at the merger time, and $\vec{J}$ is the initial total angular momentum of the system.

The relevance of precessing spinning-black-hole binaries to data analysis has been stressed in several papers using the post-Newtonian templates. "Spiky" templates have been considered to detect moderate massive galactic binaries in [60-62]. Detection and post-Newtonian dy- namics in precessing binaries have also been extensively discussed in [63-68], and the relevance to LISA observations has been discussed in [69].

\section{TECHNIQUES}

We use the Brandt-Brügmann puncture approach [27] along with the TWOPUNCTURES [70] and BAM_ELLIPTIC [71] thorns to compute initial data. In this approach the 3-metric on the initial slice has the form $\gamma_{a b}=\left(\psi_{\mathrm{BL}}+\right.$ $u)^{4} \delta_{a b}$, where $\psi_{\mathrm{BL}}$ is the Brill-Lindquist conformal factor, $\delta_{a b}$ is the Euclidean metric, and $u$ is (at least) $C^{2}$ on the punctures. The Brill-Lindquist conformal factor is given by $\psi_{\mathrm{BL}}=1+\sum_{i=1}^{n} m_{i} /\left(2 r_{i}\right)$, where $n$ is the total number of "punctures", $m_{i}$ is the mass parameter of puncture $i\left(m_{i}\right.$ is $n o t$ the horizon mass associated with puncture $i$ ), and $r_{i}$ is the coordinate distance to puncture $i$. In all cases below, we evolve data containing only two punctures with equal puncture mass parameters, and we denote this puncture mass parameter by $m_{p}$. We evolve these black-hole-binary data-sets using the LAZEV [72] implementation of the moving puncture approach [22,23]. In our version of the moving puncture approach [22] we replace the BSSN [2426] conformal exponent $\phi$, which has logarithmic singularities at the punctures, with the initially $C^{4}$ field $\chi=$ $\exp (-4 \phi)$. This new variable, along with the other BSSN variables, will remain finite provided that one uses a suitable choice for the gauge. An alternative approach uses standard finite differencing of $\phi$ [23]. Note that both approaches have been used successfully by several other groups [36,37,39,73-75].

We obtain accurate, convergent waveforms and horizon parameters by evolving this system in conjunction with a modified $1+\log$ lapse, a modified Gamma-driver shift condition [22,29], and an initial lapse $\alpha \sim \psi_{\mathrm{BL}}^{-4}$. The lapse and shift are evolved with $\left(\partial_{t}-\beta^{i} \partial_{i}\right) \alpha=-2 \alpha K, \partial_{t} \beta^{a}=$ $B^{a}$, and $\partial_{t} B^{a}=3 / 4 \partial_{t} \tilde{\Gamma}^{a}-\eta B^{a}$. These gauge conditions require careful treatment of $\chi$ near the puncture in order for the system to remain stable [22,30,75]. In Ref. [76] it was shown that this choice of gauge leads to a strongly hyperbolic evolution system provided that the shift does not become too large. For our version of the moving puncture approach, we find that the product $\alpha \tilde{A}^{i j} \partial_{j} \phi$ initially has to be $C^{4}$ on the puncture. In the spinning case, $\tilde{A}^{i j}$ is $O\left(r^{3}\right)$ on the puncture, thus requiring that $\alpha \propto r^{3}$ to maintain differentiability. We therefore choose an initial lapse $\alpha(t=0)=$ $2 /\left(1+\psi_{\mathrm{BL}}^{4}\right)$ which is $O\left(r^{4}\right)$ and $C^{4}$ on the puncture and reproduces the isotropic Schwarzschild lapse at large distances from the horizons. The initial values of $\beta^{i}$ and $B^{i}$ are set to zero.

Hannam et al. [74] examine the smoothness of the evolved fields at late times at the puncture. They find that, in the case of Schwarzschild, $\chi$ transitions from an initially $C^{4}$ field to a $C^{2}$ field at late times. Although we require that the fields be initially $C^{4}$, this late-time drop in 
smoothness does not appear to leak out of the horizon (which is consistent with the analysis in [74]).

We use a "multiple transition" fisheye transformation [30] to push the boundaries to $200 M$, while maintaining a resolution of up to $M / 30$ in the central region.

We measure the magnitude $S$ of the angular momentum of the horizons using our implementation of the algorithm detailed in [56]. The magnitude of the horizon spin is given by

$$
S=\frac{1}{8 \pi} \oint_{\mathrm{AH}}\left(\varphi^{a} R^{b} K_{a b}\right) d^{2} V
$$

where $\varphi^{a}$ is an approximate Killing vector on the horizon, $K_{a b}$ is the extrinsic curvature of the $3 \mathrm{D}$-slice, $d^{2} V$ is the natural volume element intrinsic to the horizon, and $R^{a}$ is the outward pointing unit vector normal to the horizon on the 3D-slice; the sign of $\varphi^{a}$ is chosen so that $S$ is positive. This algorithm for calculating $S$ was initially meant to be applied to the case when the individual black holes are modeled as axisymmetric isolated horizons, which is valid when the two black holes are sufficiently far away from each other. The isolated horizon formalism is generalized to the dynamical case through the notion of a dynamical horizon [52], and the formula for $S$ remains valid under this generalization.

Turning now to the direction of the spin angular momentum vector, we first note that, in general, it seems difficult to assign a unique coordinate independent 3vector $\vec{S}$ to a spinning horizon. For example, we could take a normal Kerr spacetime and slice it nonaxisymmetrically so that it becomes difficult to assign a spin 3-vector $\vec{S}$ to the black hole on these distorted 3D-slices. There is however a generalization which works. To see this, first note that every smooth cross section (with complete $S^{2}$ topology) of a Kerr horizon is axisymmetric, no matter how distorted this cross section is. This may seem somewhat surprising at first glance, but it is a straightforward consequence of the fact that the null generators of the Kerr horizon have vanishing expansion, shear and twist; the axial symmetry vector projects to a symmetry of the 2geometry of the cross section. Thus there exists a symmetry vector $\varphi^{a}$ on this cross section. The poles of the horizon are then defined to be the points where the axial symmetry vector $\varphi^{a}$ vanishes. From a spacetime perspective, the locus of points on the Kerr horizon defined by $\varphi^{a} \varphi_{a}=0$ is a coordinate and gauge independent notion. These considerations remain valid on every axisymmetric isolated horizon. As long as we have a suitable axial vector, we can similarly define the poles even for dynamical and trapping horizons. The poles exist whenever we can assign a (possibly approximate) axial symmetry vector $\varphi^{a}$ on the horizon. Of course, when the horizons become extremely distorted, it might happen that it is no longer approximately axisymmetric, or the axial vector might have more than two poles. In such extreme cases, this would not work. But we shall see through our numerical simulations that there is a significant dynamical regime where exactly 2 poles exist, and these problems do not arise.

Given the location of the two poles on the horizon, how do we assign a 3-vector to them, and thereby obtain all the components of the spin vector $\vec{S}$ ? An obvious starting point would be to use the unit normal vector $R^{a}$ at the poles. This would not give a unique answer in the absence of reflection symmetry. Alternatively, we could consider the curl of $\varphi^{a}$ suitably averaged over the horizon. However, even if we could successfully assign such a 3-vector uniquely, it is not clear in general how this vector should be compared with the spin 3-vector calculated at spatial infinity. This could be done in spacetimes with global axisymmetry, but this is not available to us in the present case. In the absence of a solution to this problem, we simply define the direction of the spin to be the Euclidean unit-norm vector tangent to the coordinate line joining the two poles. The spin-vector $\vec{S}_{\mathrm{IH}}$ is then equal to this Euclidean unit-norm vector multiplied by the isolated horizon spin obtained from Eq. (4). The definition of $\vec{S}$ might need to be further refined, however it seems to be satisfactory for our purposes. This definition of the spin vector reproduces the Bowen-York spin parameters on the initial slice, and should remain reasonable as long as the coordinates do not become too distorted. In addition to using the Killing vector $\varphi^{a}$, we also found it useful to define angular momenta with the flat space coordinate rotational killing vectors

$$
\begin{aligned}
& \varphi_{x}^{a}=\left[0,-\left(z-z_{c}\right),\left(y-y_{c}\right)\right], \\
& \varphi_{y}^{a}=\left[\left(z-z_{c}\right), 0,-\left(x-x_{c}\right)\right], \\
& \varphi_{z}^{a}=\left[-\left(y-y_{c}\right),\left(x-x_{c}\right), 0\right],
\end{aligned}
$$

where $\left(x_{c}, y_{c}, z_{c}\right)$ is the coordinate centroid of the horizon. We can then obtain the coordinate-base spin vector $\vec{S}_{\text {coord }}=\left(S_{x}, S_{y}, S_{z}\right)$ by replacing the approximate Killing vector in Eq. (4) with the three coordinate rotational vectors (i.e. $S_{i}=\frac{1}{8 \pi} \oint_{\mathrm{AH}}\left(\varphi_{i}^{a} R^{b} K_{a b}\right) \mathrm{d}^{2} V$ ). This definition of the spin direction reproduces the Bowen-York spin parameters on the initial slice as well, and produces reasonable results at later times for the gauges used here. (Of course this latter coordinate based calculation will not yield an accurate evaluation of the spin direction or magnitude for more general gauges, while the former approximate Killing vector calculation will produce accurate spin magnitudes for generic gauges.) In both cases the spin magnitude is the Euclidean norm of the 3 -vector $\vec{S}$. Note that in the former case this Euclidean norm is precisely the spin given by Eq. (4).

We solve for the approximate Killing vector field $\varphi^{a}$ on the horizon using standard spherical-polar coordinates. In these coordinates the Killing vector is obtained with highest accuracy when its poles are aligned with the coordinate 
poles of the $(\theta, \phi)$ coordinates. To make the calculation as accurate as possible, we find the minimum of $\varphi^{a} \varphi_{a}$ in the northern hemisphere and then rotate the angular coordinates so that the new north pole is aligned with the minimum of $\varphi^{a} \varphi_{a}$. We then recalculate $\varphi^{a}$ to obtain a more accurate location of the minimum and iterate until the new minimum of $\varphi^{a} \varphi_{a}$ lies on the north pole. There is a complication in the above procedure in that we cannot calculate $\varphi^{a}$ on the coordinate poles themselves (since the 2D Christoffel symbol is singular). In practice we stop iterating when the minimum of $\varphi^{a} \varphi_{a}$ lies within 2 angular grid-points of the coordinate pole. The spin direction associated with the minimum of $\varphi^{a} \varphi_{a}$ therefore cannot be obtained with higher precision than a few angular grid sizes. It might be possible to improve the accuracy by considering multiple patches on the horizon to avoid the coordinate singularity, or to use a spectral decomposition.

We found that using 160 points in the $\theta$ direction and 320 points in the $\phi$ direction provides reasonable results for the horizon spin calculation, with errors in the spin direction of about $2^{\circ}$. Adding significantly more points only increases the numerical error because the horizon algorithm itself uses far fewer points to locate the apparent horizons and the underlying numerical grid has a far coarser resolution.

The configurations discussed in this paper contain either PI-symmetry, i.e. $(x, y, z) \rightarrow(-x,-y, z)$, or paritysymmetry, i.e. $(x, y, z) \rightarrow(-x,-y,-z)$. We exploit these two symmetries to reduce the grid size by a factor of 2 . The zero-spin and (anti-)aligned spin binaries have the additional symmetry $(x, y, z) \rightarrow(x, y,-z)$. We implement the parity-symmetry boundary conditions using a locally modified version of the PI-symmetry boundary thorn kindly provided to us by Erik Schnetter. Note that behavior of the components of $g_{a b}$ and $k_{a b}$ under these symmetries can be obtained from the behavior of these components under simple reflections. Thus, $g_{x z}(x, y, z)=g_{x z}(-x,-y$, $-z)$ under parity-symmetry while $g_{x z}(x, y, z)=-g_{x z}(-x$, $-y, z)$ under PI-symmetry. The Einstein equations preserve these symmetries. In the case of spinning, equalmass binaries, parity-symmetry requires that the two spins be equal in magnitude and parallel (i.e. the spin-vector behaves as a pseudovector).

\section{INITIAL CONFIGURATIONS}

We study two configurations of nonaligned-spin binaries with parallel spins (equal in magnitude) that exhibit spin and orbital-plane precession, as well as spin-flips of the remnant horizon spin with respect to the individual horizon spins. We choose configurations where the binary separation is small enough that the spin-orbit coupling is large, but large enough that the binaries complete at least $\sim 1.75$ orbits before merging. The first configuration, which we denote with SP3 starts with the spins aligned along the initial orbital plane. This can be interpreted as a binary in which one black hole orbits about the pole of the second black hole. The second configuration, which we denote with SP4, starts with the spins pointing $45^{\circ}$ above the initial orbital plane, corresponding to a binary in which infall occurs initially along a plane tilted with respect to both spins. In both cases the masses and spins of the two holes are equal (i.e. spins parallel and equal in magnitude). Setting the two masses and spins equal ensures that the system is parity-symmetric, but still is generic enough to display both spin and orbital plane precession as well as a spin-flip in the direction of the orbital angular momentum. The initial data parameters for these two configurations, which were obtained using the 3PN equations of motion, are given in Table I. We also report the initial-data parameters for the previously studied aligned $(\mathrm{S}++, \mathrm{SC})$, antialigned $(\mathrm{S}--)$, and nonspinning binaries $(S 0)$. The $\mathrm{PN}$ data provides the puncture location, momenta, and spins. We complete the data by choosing puncture mass parameters (equal for the two punctures) such that the total ADM mass of the system is 1 .

\section{RESULTS}

We evolved the SP3 configuration using central resolutions of $h=M / 22.5, h=M / 25$, and $h=M / 30$; with grid-sizes of $576^{2} \times 288,640^{2} \times 320$, and $768^{2} \times 384$ respectively. We used "multiple transition" fisheye transformation [30] to place the outer boundaries at 200M; far enough away that boundary effects do not interfere with the orbital dynamics of the system. In addition, we also evolved the SP4 configuration with a central resolution of

TABLE I. Initial data for quasicircular, equal-mass black-hole binaries. The binaries have an ADM mass of (1.0000 \pm 0.0005$) M$, with orbital frequency $M \Omega$ fixed to 0.0500 , and initial proper separations $l$. The punctures are located at $( \pm X, 0,0)$, with mass parameter $m_{p}$, momentum $(0, \pm P, 0)$, spin angular momentum $\left(0, S_{y}, S_{z}\right)$, and specific spin $S / m^{2}$ ( $m$ is the horizon mass).

\begin{tabular}{|c|c|c|c|c|c|c|c|c|}
\hline Name & $S_{y} / M^{2}$ & $S_{z} / M^{2}$ & $X / M$ & $P / M$ & $J / M^{2}$ & $S / m^{2}$ & $l / M$ & $m_{p} / M$ \\
\hline SP3 & 0.128725 & 0 & 3.276347 & 0.133587 & 0.91243 & 0.5013 & 10.20 & 0.43025 \\
\hline SP4 & 0.091198 & 0.091198 & 3.179908 & 0.1314406 & 1.03454 & 0.5007 & 9.94 & 0.43037 \\
\hline S0 & 0.0 & 0.0 & 3.280 & 0.1336 & 0.876 & 0.0 & 10.01 & 0.4848 \\
\hline$S C$ & 0.0 & 0.025757 & 3.2534 & 0.1330 & 0.917 & 0.1001 & 9.93 & 0.4831 \\
\hline $\mathrm{S}++$ & 0.0 & 0.1939 & 3.0595 & 0.1291 & 1.1778 & 0.757 & 9.27 & 0.3344 \\
\hline$S--$ & 0.0 & -0.1924 & 3.465 & 0.1382 & 0.5729 & -0.757 & 10.3 & 0.3344 \\
\hline
\end{tabular}




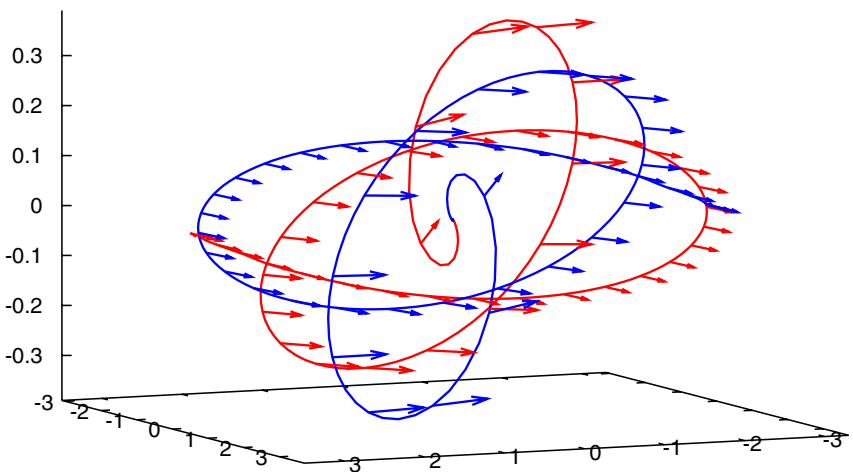

FIG. 1 (color online). The puncture trajectories along with spin direction (every $4 M$ ) for the SP3 configuration for the $M / 30$ resolution run. The spins are initially aligned along the $y$-axis, but rotate by $\sim 90^{\circ}$ during the 1.25 last orbits and also acquire a non-negligible $z$-component. Note that the $z$-scale is $1 / 10$ th the $x$ and $y$ scale.

$h=M / 25$, a grid size of $640^{2} \times 320$, and outer boundary at $200 M$.

Figs. 1 and 2 show the puncture trajectory and horizonspin direction along this track for the SP3 configuration (the latter suppressing the $z$-direction). Note that the scale of the $z$-axis in Fig. 1 is $1 / 10$ th that of the $x$ and $y$ axes.

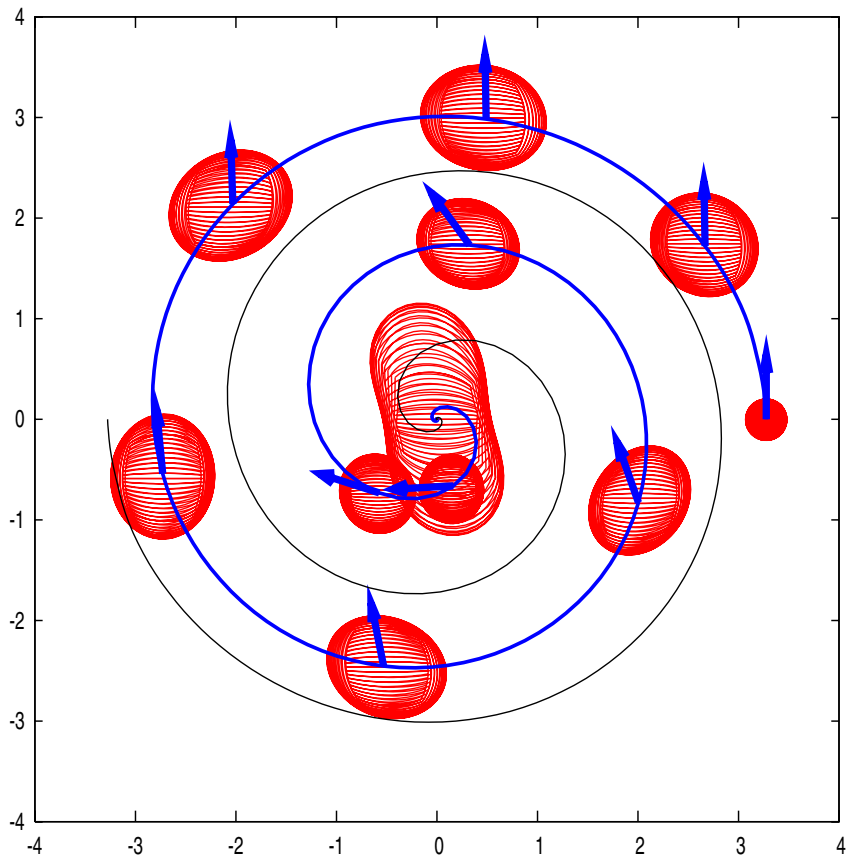

FIG. 2 (color online). The projection of puncture trajectories and spin for the SP3 configuration onto the $x y$ plane along with the individual apparent horizons for the $M / 30$ run. The horizons and spins are shown at $t=0,20 M, 40 M, \ldots, 160 M, 164 M$. The first common horizon (also shown) formed at $t=164.2 M$. The spins are initially aligned along the $y$-axis but rotate by $\sim 90^{\circ}$ during the last 1.25 orbits. The spin of the second black hole (not shown) is equal to the first.
From the plots one can clearly see the orbital plane precess out of the equatorial plane, as well as the spin axis rotating by approximately $90^{\circ}$ in the $x y$ plane during the course of the merger. The spins are initially aligned along the $y$-axis, but at merger they show both a significant $z$-component and an approximate $90^{\circ}$ rotation to the $-x$-axis. The individual horizon spins at the merger are $\vec{S}_{\text {coord }}=$ $(-0.121 \pm 0.002,-0.007 \pm 0.003,0.037 \pm 0.003) \quad$ (we use the coordinate based measure of the spin at the merger because the calculation of $S_{\mathrm{IH}}$ is not accurate when the black holes are this close together; see comments below). Hence the total precession angle for the SP3 configuration is $\Theta_{p}=98^{\circ}$. Note that there is no discernible correlation between the orientation of the projected horizon and the projected spin direction.

In Fig. 3 we show the coordinate $\left(\vec{S}_{\text {coord }}\right)$ and Killing vector based $\left(\vec{S}_{\mathrm{IH}}\right)$ calculation of the spin components versus time. $\vec{S}_{\mathrm{IH}}$ displays a step-function-like behavior due to the difficulty in finding the poles (i.e. the zeroes of $\varphi^{a} \varphi_{a}$ ) in the Killing vector accurately. As discussed above, the Killing vector calculation is most accurate when its poles are located at the coordinate poles of the $(\theta, \phi)$ coordinates on the horizon. However, the difficulty in

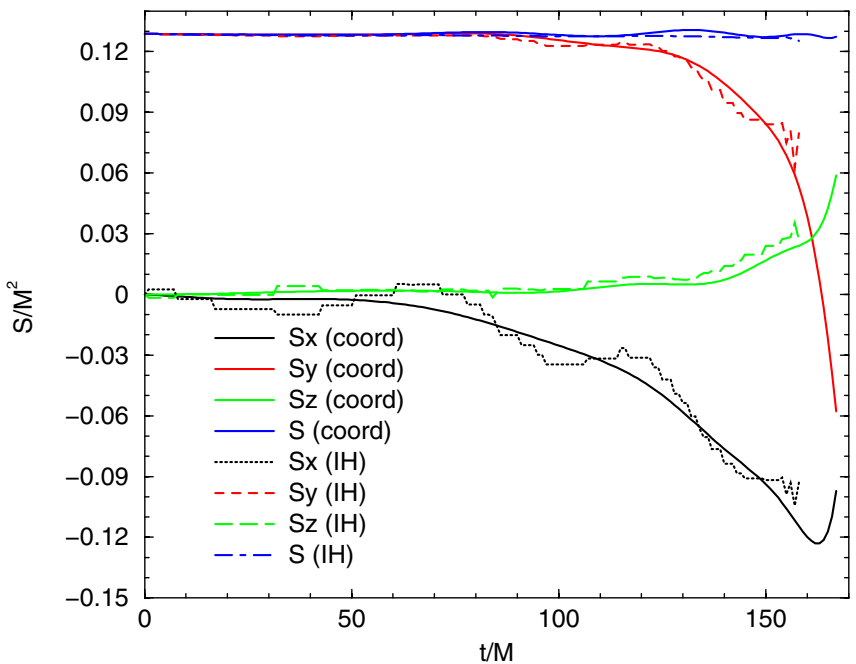

FIG. 3 (color online). The spin components and magnitude versus time for the SP3 configuration as calculated using the coordinate rotational vectors (coord) and the poles of the approximate Killing vector $(\mathrm{IH})$ for the $M / 30$ resolution. The calculation of the approximate Killing vector breaks down near the merger (which occurs at $t=164.2$ ), but the purely coordinate-based calculation continues to produce reasonable results. Note that the direction obtained from the Killing vector oscillates about the coordinate based direction. Also note the spin has just rotated by $90^{\circ}$ in the $x y$ plane at the time of merger. The spin magnitude remains essentially constant throughout the merger phase. The magnitude of the spin calculated from the Killing vector is coordinate invariant and, unlike the spin direction, is expected to be more accurate than the coordinate-based calculation. 
calculating the Killing vector itself near the coordinate poles introduces an uncertainty in the location of the Killing vector poles. We also stress that the direction associated with the location of the two poles of the Killing vector is coordinate dependent. From the figure we see that the $x$ - and $y$-components of $\vec{S}_{\mathrm{IH}}$ oscillates about the much more regular $x$ and $y$ components of $\vec{S}_{\text {coord }}$, while the $z$-component of $\vec{S}_{\mathrm{IH}}$ is consistently larger than the $z$-component of $\vec{S}_{\text {coord }}$. The calculation of $S_{\text {IH }}$ (and hence $\vec{S}_{\text {IH }}$ ) breaks down prior to the merger when the horizons get too close (and hence the mutual tidal distortions destroy the approximate axial symmetry). $\vec{S}_{\text {coord }}$, however, continues to produce reasonable results through the merger. Thus, it is $\vec{S}_{\text {coord }}$ that shows the clear rotation of the spin from the $y$-axis to the $x$-axis at the merger. Note that the uncertainties in the spin directions do not correspond to uncertainties in the magnitudes of the spin. For the Killing vector based calculation $\vec{S}_{\mathrm{IH}}$, it is the spin magnitude that is determined with high accuracy. Figure 4 shows between third and fourth-order convergence of the components of $\vec{S}_{\text {coord }}$ from the three resolutions (the third-order error may be due to third-order errors leaking out of the puncture as well as third-order errors from the horizon calculations), while Fig. 5 shows the value of the $z$-component of the specific spin $S_{z} / m^{2}$ (where $m$ is the horizon mass) based on the $z$-component of $\vec{S}_{\mathrm{IH}}$ for the three resolutions. In this latter figure the curves have been translated. (A convergence plot of $\vec{S}_{\mathrm{IH}}$ would not be meaningful because the size of the step discontinuities in $\vec{S}_{\mathrm{IH}}$ are larger than the differences in the

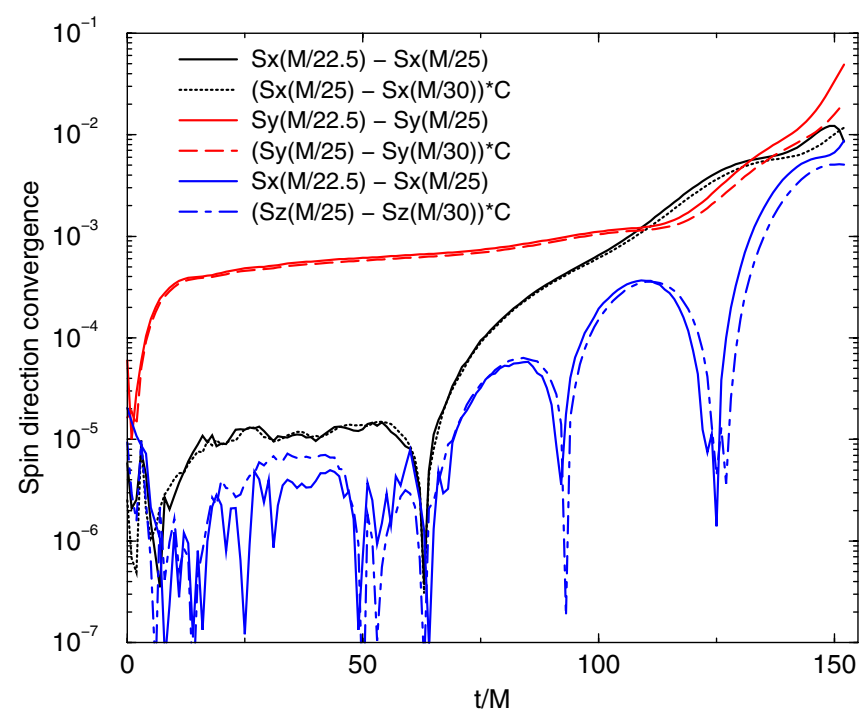

FIG. 4 (color online). A convergence plot of the coordinatebase $\vec{S}_{\text {coord }}$ calculation for the SP3 configuration. Note that for our choice of resolutions, third-order convergence is demonstrated by $S(M / 22.5)-S(M / 25)=(S(M / 25)-S(M / 30)) C$, where $C=0.88$. The spin is initially third-order convergent, with higher order-convergence apparent at later times.

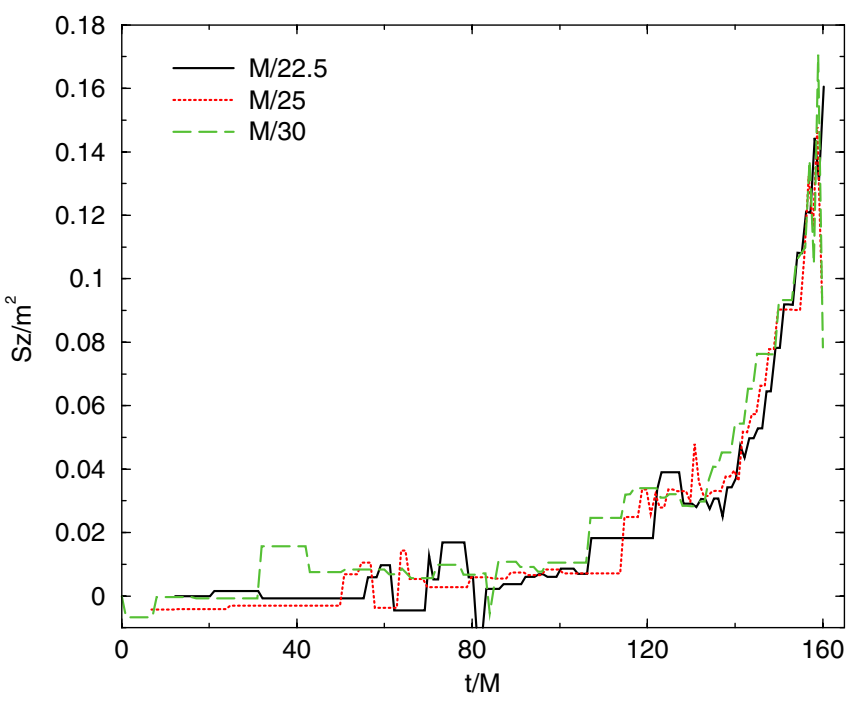

FIG. 5 (color online). The $z$-component of the Killing vector based calculation of the spin rescaled by the square of the horizon mass for the three resolutions. The curves have been translated by a distance equal to the difference in merger times of the $M / 22.5$ and $M / 25$ runs with the merger time of the $M / 30$ run. In this configuration, unlike the previously studied alignedspin binary, the "spin-up" appears to be large. However, in this case the "spin-up" is actually the rotation of the nearly-constant spin-vector towards the $z$-axis, rather than an increase in the spin magnitude.

spin direction with resolution.) For the $z$-component of the spin, we expect that, given the lack of significant oscillations plaguing the $x$ and $y$ components, $\vec{S}_{\mathrm{IH}}$ gives a better measurement than the more highly coordinate-dependent $\vec{S}_{\text {coord }}$. The spin-up of the z-component of the specific spin by 0.16 is 10 times larger than the analogous spin-ups of about 0.01 seen in the zero-spin (S0) and aligned-spin (SC) configurations (see [34]). However, as can be seen in Fig. 3, the spin magnitude does not increase significantly. Thus this spin-up in the $z$-direction is not equivalent to the spin-up observed in the case of the aligned-spin and nonspinning binaries. In those cases the spin-up involved an increase in the spin magnitude, while here it primarily involves a rotation of the spin vector out of the $x y$ plane. This rotation of the spin out of the $x y$ plane follows the post-Newtonian predictions of Eq. (1).

In Fig. 6 we plot the $x$ and $y$ components of the spin as a function of the $z$ component for both the post-Newtonian predicted spins (using numerical tracks) and the numerically determined spins. Plotting the data in this manner removes the ambiguity of assigning the appropriate postNewtonian time to the numerical time coordinate on the horizon. The qualitative behavior of the spin in our numerical simulation is consistent with the post-Newtonian spin for most of the evolution (smaller values of $S_{z}$ ).

The puncture trajectories are third-order convergent as is demonstrated in Fig. 7. The $x$-component of the track appears to show poorer convergence between $t=10 \mathrm{M}$ 


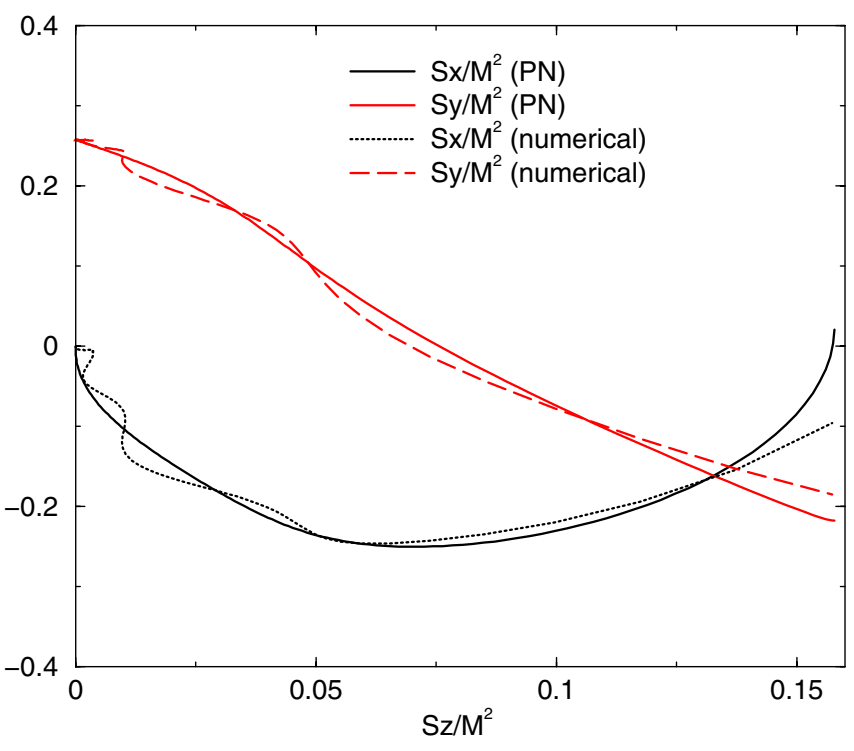

FIG. 6 (color online). The post-Newtonian and numerical $S_{x}$ and $S_{y}$ components of the spin of the individual horizons for the SP3 configuration as functions of the $S_{z}$ component of the spin (which increases monotonically in time prior to the merger). Note the very reasonable agreement for most of the evolution. The plot terminates at the merger.

and $t=60 \mathrm{M}$ but this is likely due to the coarseness of the grid. Note that the trajectories are calculated by integrating $\partial_{t} x^{a}=-\beta^{a}$ [22] at the location of the punctures. The curve is expected to converge to lower order because the shift is not smooth on the puncture (see [74] for a discus-

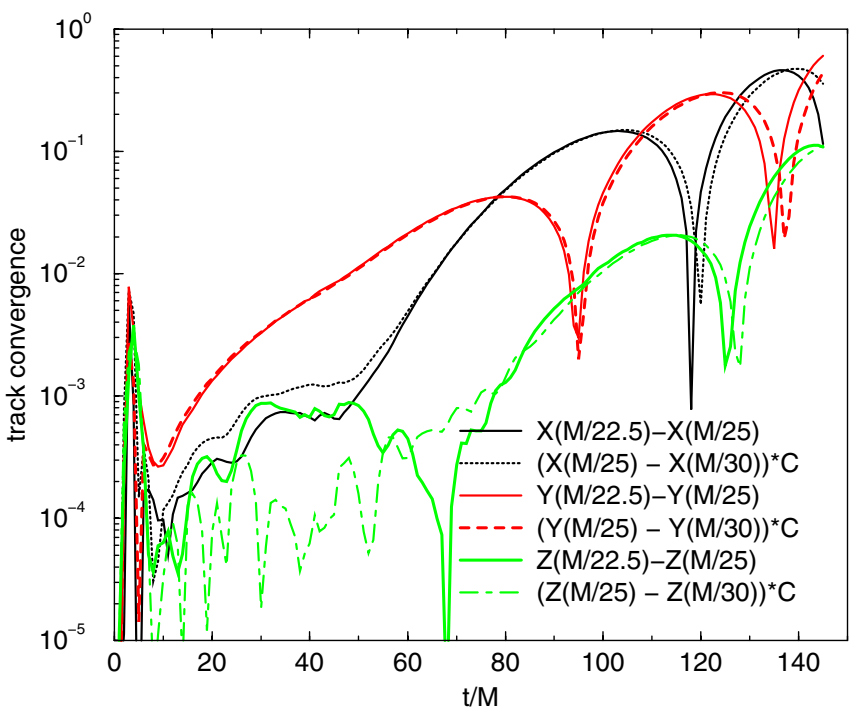

FIG. 7 (color online). The difference in track locations between the $M / 22.5$ and $M / 25$ runs as well as the difference in track locations between the $M / 25$ and $M / 30$ runs. The latter difference has been rescaled by $C=0.88$ to demonstrate thirdorder convergence. The reduced order of convergence for $X$ between $t=10 \mathrm{M}$ and $t=60 \mathrm{M}$ is likely due to the coarseness of the grid. sion on the behavior of the evolved fields at the punctures). However, as is shown below, the constraint violations also show third-order convergence, which indicates that lowerorder convergent effects leak out of the puncture. These lower-order errors are likely not observed in the waveform (see below) because of a larger fourth-order error term dominating the third-order error terms at these resolutions.

The final remnant horizon for the SP3 configuration has mass $M_{\mathcal{H}} / M=0.9613 \pm 0.0007$ with specific spin $S / M_{\mathcal{H}}^{2}=0.7215 \pm 0.0003$. The remnant spin components calculated from the approximate Killing vector are $\vec{S}_{\mathrm{IH}} / M^{2}=(-0.045 \pm 0.001,0.199 \pm 0.003,0.638 \pm 0.003)$ with magnitude $S_{\mathrm{IH}} / M^{2}=0.669 \pm 0.001$. The total ADM mass and angular momentum (i.e. initial mass and angular momentum) of the system are $M_{\mathrm{ADM}} / M=1.00000 \pm$ 0.00005 and $\vec{J}_{\mathrm{ADM}} / M^{2}=(0,0.257450,0.875352)$. Hence $(3.87 \pm 0.07) \%$ of the mass and $(23.6 \pm 0.1) \%$ of the angular momentum were converted into radiation, with $\delta J_{z} / M^{2}=(0.237 \pm 0.003)$. The system gained net angular momentum in the $x$-direction but lost $(22.7 \pm 0.4) \%$ of its angular momentum in the $y$-direction and $(27.1 \pm$ $0.3) \%$ of its angular momentum in the $z$-direction. Thus the binary preferentially radiated angular momentum in the direction of the initial orbital angular momentum. We also obtained estimates of the radiated mass and $z$-component of the angular momentum from $\psi_{4}$ of $(3.8 \pm 0.1) \%$ and $(0.24 \pm 0.02) M^{2}$ respectively, in excellent agreement with the results from the remnant horizon parameters. (The errors in the radiated mass and angular momentum from the waveform are relatively large due to boundary reflection contaminating the late-time waveform.) Note that the excellent agreement in $\delta J_{z}$ between the final horizon direction measurement and the radiated $z$-component of the angular momentum indicates that we obtain the final horizon spin magnitude and direction to within the expect $2^{\circ}$ accuracy. For comparison we also give the remnant spin direction calculated using the coordinate rotation vectors $\quad \vec{S}_{\text {coord }} / M^{2}=(-0.033 \pm 0.002,0.190 \pm 0.001$, $0.6395 \pm 0.0003$ ) (where the errors are a measure of the flatness of the components of $\vec{S}_{\text {coord }}$ versus time). The corresponding spin magnitude $S_{\text {coord }} / M^{2}=0.668 \pm$ 0.002 agrees with the norm $S_{\mathrm{IH}}$ and the directions agree to within $1.3^{\circ}$ (the expected error in the direction determination is $\sim 2^{\circ}$ ).

We measure the angle of the spin-flip both with respect to the initial individual horizon spins and the individual horizon spins at the merger. For the SP3 configurations these angles are $72^{\circ}$ and $71^{\circ}$, respectively. In Fig. 8 we show the spin direction of the individual horizons and spin direction of the remnant horizon. The smooth precession and discontinuous flip are apparent. Note that the spin flip, unlike the spin precession, cannot be modeled accurately by a post-Newtonian expansion due to the highly nonlinear merger process that converts roughly $25 \%$ of the initial total angular momentum into gravitational radiation. The 


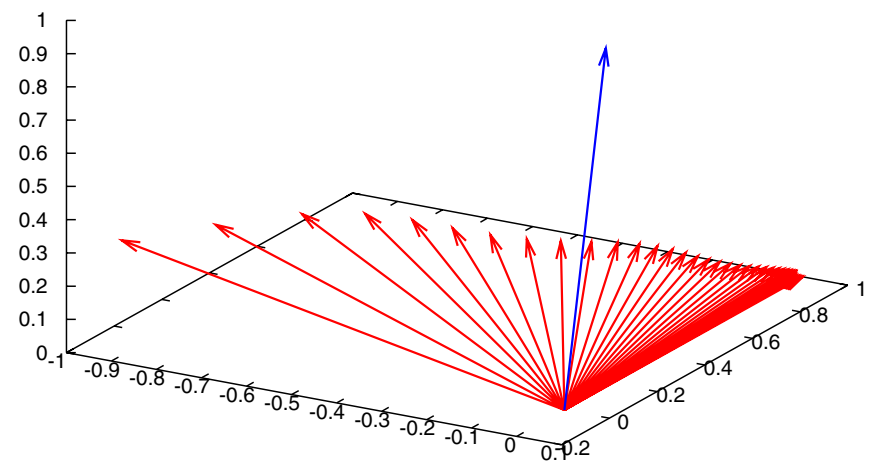

FIG. 8 (color online). The spin direction of the individual horizons every $4 M$ during the spin-precession phase and the final horizon spin direction for the SP3 configuration. The arrows indicate the spin direction only, not the magnitude. Note the continuous change in the spin direction during the precession stage and the discontinuous jump (or flip) to the remnant spin direction.

final spin direction for the SP3 configuration is rotated by $4.0^{\circ}$ with respect to the initial ADM angular momentum. This rotation, though small, is larger than the expected error in our spin direction algorithm, indicating that there is a small net change in the direction of the angular momentum.

The spin direction and puncture trajectories are coordinate dependent measures of precession. The waveform, on the other hand, should provide a coordinate independent measure of the precession. To show the effect of precession on the waveform we examine the $(\ell=2, m=1)$ mode. This mode vanishes identically for the zero-spin, alignedspin, and antialigned spin cases previously studied (i.e. S0,

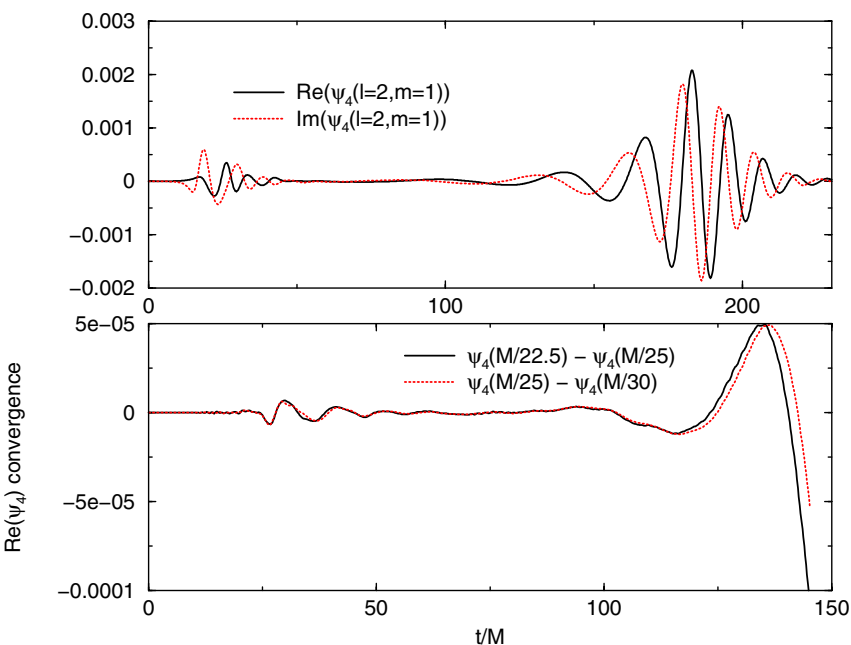

FIG. 9 (color online). The real and imaginary parts of the $(\ell=$ $2, m=1)$ mode of $\psi_{4}$ for the SP3 configuration with resolution $h=M / 25$ (upper panel), as well as a convergence plot of this waveform (lower panel). Note that the waveform is fourth-order convergent (as is evident by the agreement of the two differences).
$\mathrm{SC}, \mathrm{S}++, \mathrm{S}--)[22,30,34,35]$. In Fig. 9 we show the $(\ell=2, m=1)$ mode at an extraction radius of $r=15 \mathrm{M}$ as well as a convergence plot of this mode (showing fourthorder convergence). The contributions of the $(\ell=2, m=$ $\pm 1)$ modes to the radiated mass are smaller than the contributions of the dominant $(\ell=2, m= \pm 2)$ modes by a factor of $\sim 20$, while the contributions of the $(\ell=$ $2, m= \pm 1)$ modes to the radiated angular momentum are smaller than the contributions of the dominant $(\ell=2, m=$ \pm 2 ) modes by a factor of $\sim 80$.

We next examine how the results change when we set the initial spins closer to the $z$-axis. The SP4 configuration has the same total spin as the SP3 configuration but at an angle of $45^{\circ}$ with respect to the orbital plane. This rotation of the spin has two significant effects. First, the SP4 configuration has a significant spin in the same direction as the orbital angular momentum, and from our previous results [34,35] we expect that the binary merger will be delayed due to the resulting spin-orbit repulsive effect. Second, the amplitude of the orbital plane precession will be reduced (i.e. there is no orbital plane precession if the spins are rotated $90^{\circ}$ with respect to the orbital plane, and the amount of precession should vary smoothly with angle). In Fig. 10 we show the $z$-component of the first puncture trajectory versus time for SP3 and SP4, where the latter has been rescaled by $\sqrt{2}$. Note that at early times the rescaled tracks agree perfectly. Thus, for a given magnitude of the spin, the orbital plane precession has a $\sin \vartheta$ dependence, where $\vartheta$ is the angle between the spin and orbital angular momentum. At later times, the spin-orbit coupling induced delay in the merger becomes evident and the two tracks no longer agree.

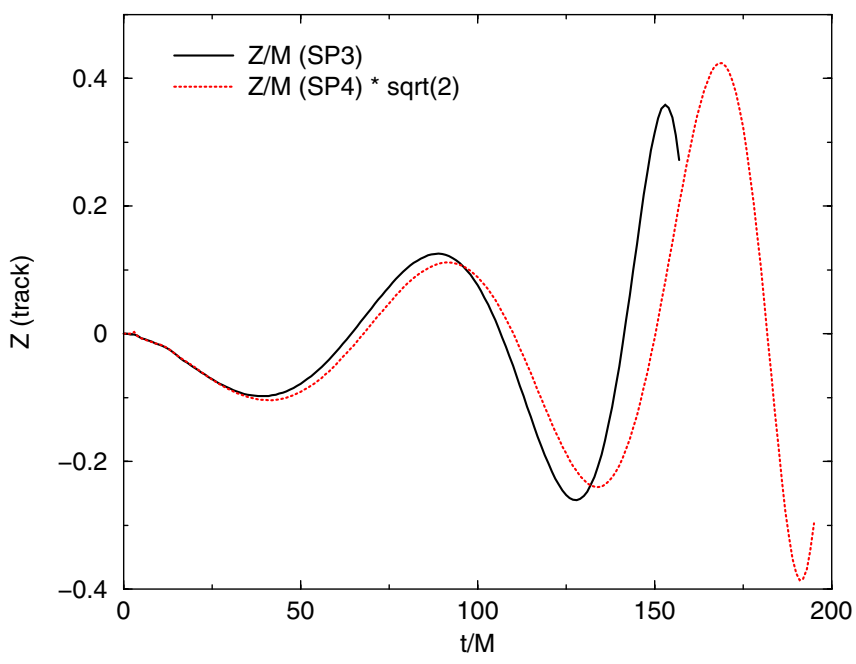

FIG. 10 (color online). The $z$-component of the puncture trajectory (for the puncture initially located at $x>0$ ) versus time for the SP3 and SP4 configurations with resolution $h=M / 25$. Note that after rescaling by $\sqrt{2}$ the two trajectories agree at early times. At later times the spin-orbit coupling in the partially aligned case delays the merger; causing the two trajectories to diverge. Both curves terminate at their respective merger times. 
MANUELA CAMPANELLI et al.

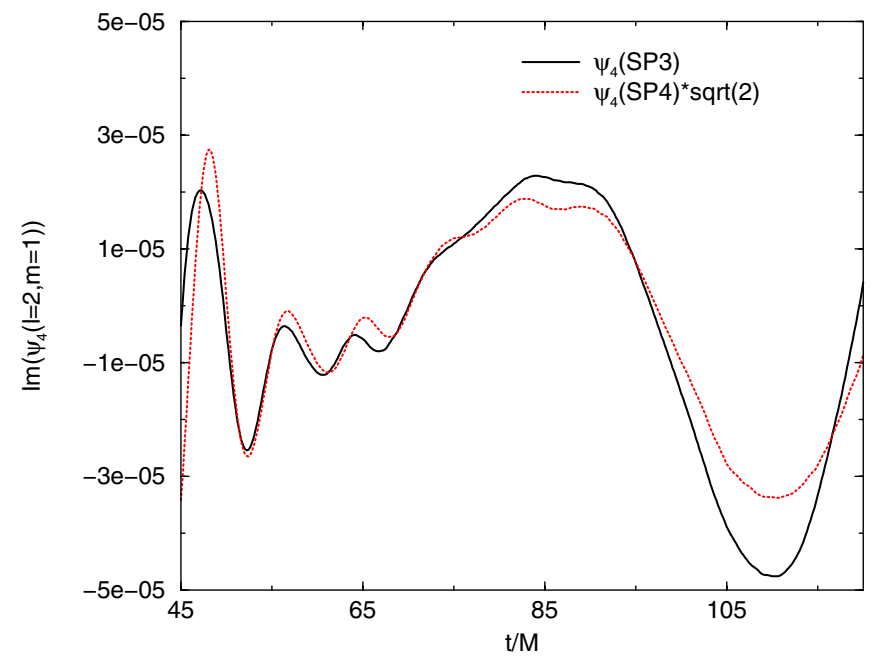

FIG. 11 (color online). The imaginary part of the $(\ell=2, m=$ 1) component of $\psi_{4}$ for the SP3 and SP4 configurations extracted at $r=15 M$ with a central resolution of $M / 25$. Note that between $t=50$ and $t=75$ the two waveforms scale with $\sin \vartheta$ as is evident by the good agreement between the two waveform after rescaling the SP4 waveform by $\sqrt{2}$ [i.e. $1 / \sin (\pi / 4)$ ]. However, this scaling breaks down at later times $(t>85 M)$ due to the differences in orbital decay arising from the increased stability of the SP4 configuration. This scaling also breaks down at early times because of the nonphysical initial data radiation pulse.

Comparing the waveforms from the SP3 and SP4 configurations is complicated by the fact that the initial data burst masks the early-time behavior. Nevertheless, there is a small region just after the initial pulse leaves the system (see Fig. 11) where it is evident that the $(\ell=2, m=1)$ mode scales with $\sin \vartheta$. However, at later times the differences in the orbital dynamics destroys this scaling.

In Figs. 12-14 we show the spin magnitude and direction for the SP4 configuration, as well as the 3D puncture trajectories and spins and a projection of the trajectories and spin direction onto the $x y$ plane. Because of the increased stability of aligned spin binaries, this configuration completes $2 \frac{3}{8}$ orbits prior to merger (compared to $1 \frac{3}{4}$ for SP3). Consequently spin-precession rotates the spin vector by an additional $45^{\circ}$ compared to the SP3 configuration. In this case the Killing vector based calculation of the spin remains accurate long enough that the spinprecession rotation beyond $90^{\circ}$ (i.e. the local minimum in $S_{x}$ is observed in $\vec{S}_{\mathrm{IH}}$ as well as $\vec{S}_{\text {coord }}$ ). Note that, once again, there is a significant spin-up in the $z$-direction caused by a rotation of the spin vector towards the $z$-axis (rather than a net increase in the spin amplitude), and that there is no discernible correlation between the projected horizon orientation (i.e. the orientation of the semimajor axis) and the projected spin direction. The individual horizon spins at the merger are $\vec{S}_{\text {coord }}=(-0.033 \pm$ $0.005,-0.041 \pm 0.003,0.114 \pm 0.001)$. Hence the total precession angle for the SP3 configuration is $\Theta_{p}=151^{\circ}$.
PHYSICAL REVIEW D 75, 064030 (2007)

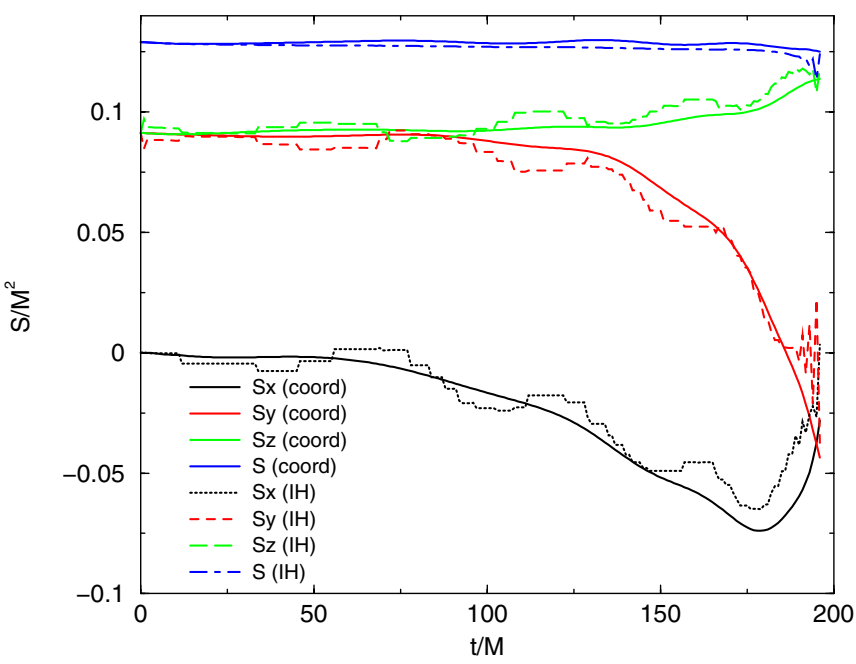

FIG. 12 (color online). The spin components and magnitude of the individual horizons (the two horizons have equal spins) for the SP4 configuration up to merger (with resolution $h=M / 25$ ). Note that in this configuration the spins rotate by $135^{\circ}$ in the $x y$ plane prior to merger. Also note that the significant $z$-direction spin-up is once again caused by a rotation further out of the $x y$ plane. Once again the Killing vector determination for the spin direction oscillates about the coordinate definition for the spin direction. The approximate Killing vector calculation begins to break down at around $t=180 \mathrm{M}$.

The remnant horizon for the SP4 run formed just as the boundary reflections began to contaminate the interior. Consequently the error bounds for the mass and spin of the remnant are higher for SP4 than SP3. The final remnant mass is $M_{\mathcal{H}}=(0.9524 \pm 0.0002) M$ with a spin parameter of $S / M_{\mathcal{H}}^{2}=0.805 \pm 0.002$. The spin components are $\vec{S}_{\text {coord }} / M^{2}=(-0.020 \pm 0.003,0.121 \pm 0.002$, $0.720 \pm 0.002)$, where we used the coordinate-base definition to calculate $\vec{S}$. (The Killing vector based calculation of the spin could not be obtained accurately because the system became approximately axisymmetric after the boundary errors affected the remnant spin parameters.)

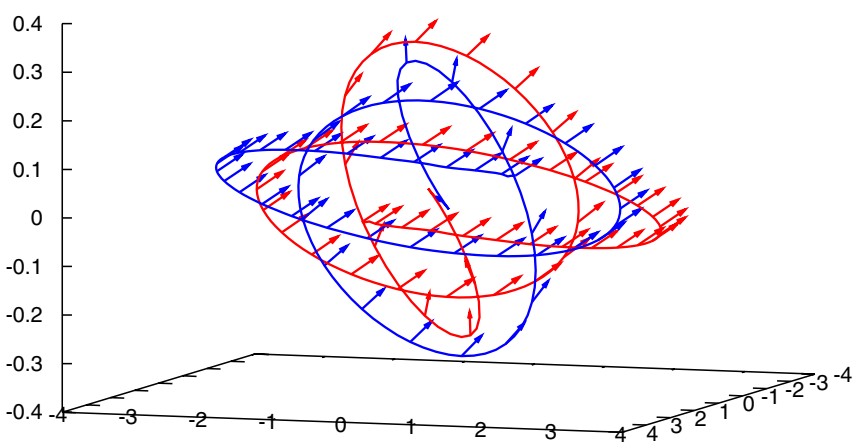

FIG. 13 (color online). The puncture trajectories and horizon spin direction (shown every $4 M$ until merger) for the SP4 configuration. Note that the $z$-scale is $1 / 10$ th that of the $x$ and $y$ scales. 


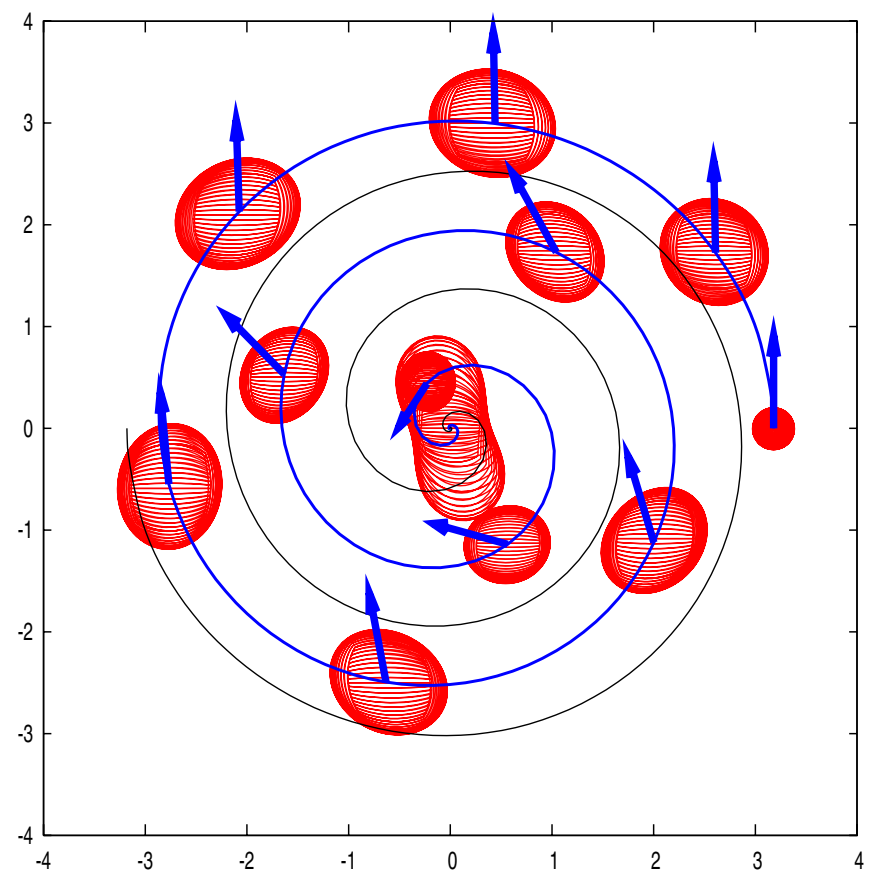

FIG. 14 (color online). The $x y$ plane projection of the orbital track, apparent horizons, and spin direction for the SP4 configuration up to merger. The horizons are given at $t=0,20 M$, $40 M, \ldots, 180 M, 196 M$. The common horizon formed at $t=$ $195.4 M$. The projected spin vector decreases in magnitude at late time due to the spin rotating further out of the $x y$ plane. Note that the spin direction precesses by $135^{\circ}$ in the $x y$ plane during the merger. The spin of the second black hole (not shown) is equal to the first.

The initial ADM mass and angular momentum for this system were $M_{\mathrm{ADM}} / M=1.00000 \pm 0.00005$ and $\vec{J}_{\mathrm{ADM}} / M^{2}=(0,0.182396,1.01833)$. Hence, $\quad(4.76 \pm$ $0.02) \%$ of the mass and $(29.4 \pm 0.2) \%$ of the angular momentum were converted into gravitational radiation. The system gained net angular momentum in the $-x$ direction, while losing $(34 \pm 1) \%$ and $(29.3 \pm 0.2) \%$ of its angular momentum in the $y$ and $z$ directions, respectively. It thus appears that this configuration preferentially radiates angular momentum in the orbital plane. However, we caution the reader that the errors quoted for the SP4 configuration for the mass and spin do not take into account either possible boundary effects or finite-difference truncation errors. The effect of the radiation on the angular momentum direction is small, with the SP4 spin direction rotated by only $1.5^{\circ}$ with respect to the initial ADM angular momentum.

The spin-flip angle of the remnant spin with respect to the initial individual spins and the individual spins at merger are $35^{\circ}$ and $32^{\circ}$, respectively. In Fig. 15 we show the spin direction of the individual horizons and spin direction of the remnant horizon. The smooth precession and discontinuous flip are apparent. Note that in this case boundary reflections contaminate the waveform at large $r$ prior to the merger. Consequently we do not obtain reliable

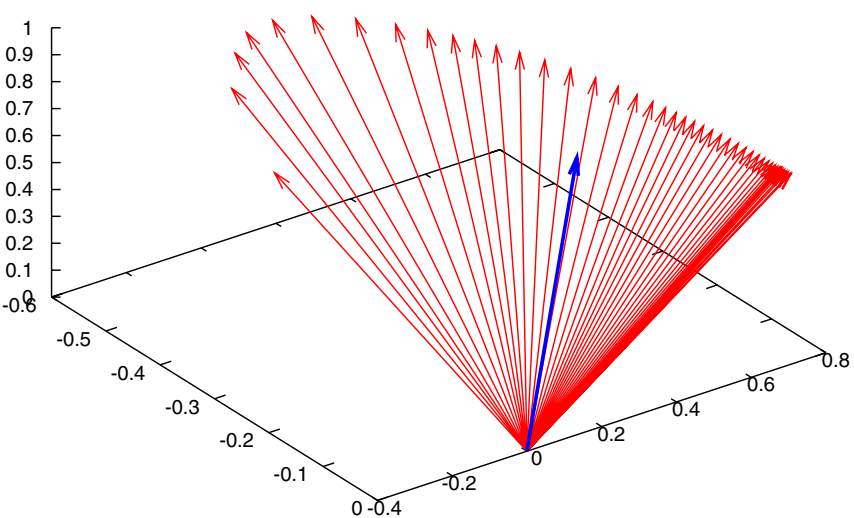

FIG. 15 (color online). The spin direction of the individual horizons every $4 M$ during the spin-precession phase and the final horizon spin direction for the SP4 configuration. The arrows indicate the spin direction only, not the magnitude. Note the continuous change in the spin direction during the precession stage and the discontinuous jump (or flip) to the remnant spin direction.

measurements for the radiated mass and angular momentum from the waveform.

As was mentioned above, the calculation of the spin direction is coordinate dependent. Nevertheless, these particular coordinates show remarkable agreement between the puncture trajectories and the waveform. In Fig. 16 we show the orbital part of the $(\ell=2, m=2)$ component of $\psi_{4}$ extracted at $r=10 M$, where we translated the SP4

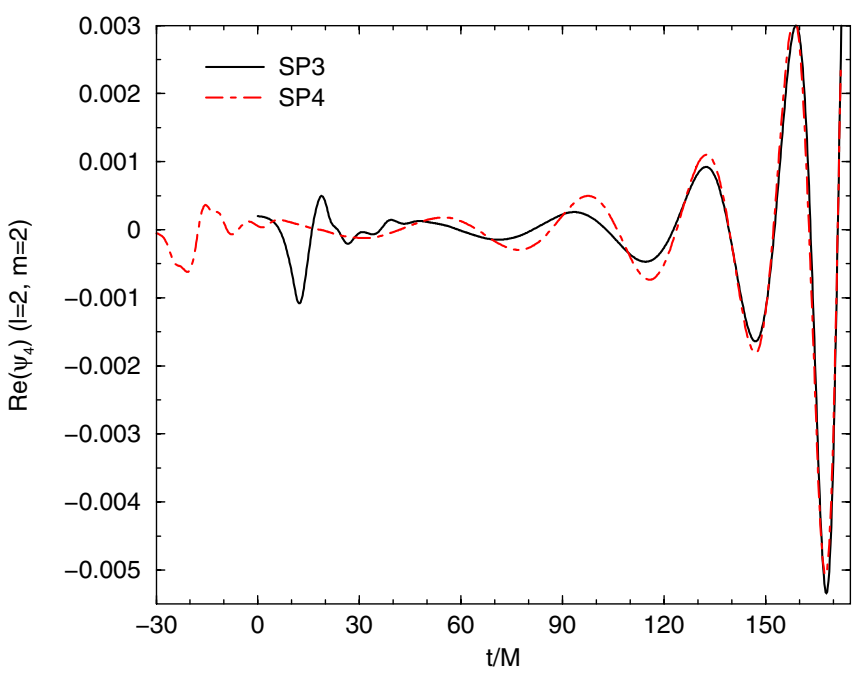

FIG. 16 (color online). The real part of the premerger $(\ell=$ $2, m=2$ ) component of $\psi_{4}$ extracted at $r=10 M$. The SP4 waveform has been translated by $37 M$ and multiplied by a constant phase factor prior to taking the real part. The plunge part of the waveform begins roughly at $t=168 M$. Thus there are 3.5 cycles of orbital radiation prior to the plunge (but after the initial data pulse) for SP3 and 4.5 for SP4. This number of cycles match the number of orbits in Figs. 2 and 14. The initial data pulse appears to mask an additional $1 / 2$ cycle of orbital motion. 
waveform and multiplied by a constant phase in such a way that plunge part of the waveforms agree [32]. Note that the SP3 configuration shows $\sim 4$ cycles of orbital motion prior to the plunge (i.e. the last trough in the plot), while the SP4 configuration shows $\sim 5$ cycles. Thus we expect that the SP4 track should contain approximately one-half of an orbit more than the SP3 configuration. Although there is an uncertainty in the exact location of the beginning of the "plunge" waveform in the figure, its approximate location will be given by the formation time of the common horizon at this resolution plus the coordinate distance to the extraction sphere (here we identify the start of the plunge with the first trough located at $t=168=T_{\mathrm{CAH}}+10 M$ ), the number of cycles in both configurations prior to the last peak shown is consistent with $1 / 4$ of an orbit more than the number of orbits (i.e. 1.75 and 2.25 , respectively) observed in Figs. 2 and 14. Interestingly, the number of cycles after the initial pulse of radiation ( 3.5 and 4.5 , respectively) is in excellent agreement with the number of orbits observed in the puncture trajectories. Thus these coordinates appear to reasonably reproduce the orbital dynamics of the binary. This fidelity by which the coordinates reproduce the merger dynamics, and the relatively good agreement between the two measurements of the spin direction, is the motivation for using these coordinate dependent measurements to measure the spin direction. In addition, the very good agreement for the radiated $z$-component of the angular momentum based on the difference between the remnant $\vec{S}_{\mathrm{IH}}$ and $\vec{J}_{\mathrm{ADM}}$ for SP3 and the waveform-based calculation, indicates that this method provides an accurate

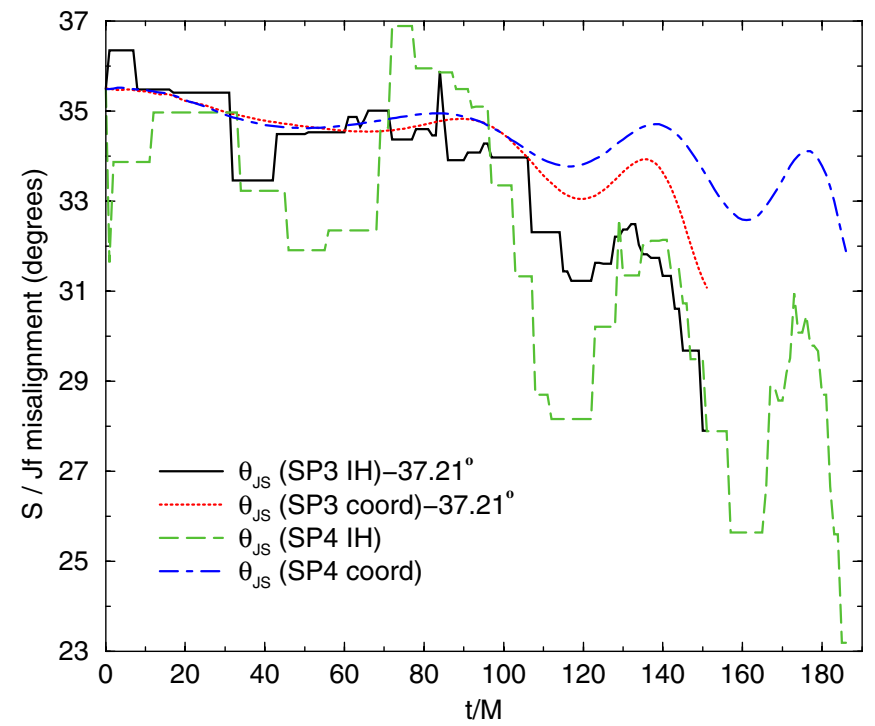

FIG. 17 (color online). The angle in degrees between the individual apparent horizon spins and the final total angular momentum for the SP3 and SP4 configuration. The SP3 angles have been translated by $-37.21^{\circ}$ so that the translated SP3 and SP4 angles agree at $t=0$. The curves terminate at the point when the isolated horizon algorithm becomes inaccurate. measurement of the remnant spin direction and magnitude. We expect the method to provide more accurate results for the remnant horizon than the individual horizon because the remnant spacetime is axisymmetric, and, as pointed out above, there is a natural way to assign a spin direction to horizons when the spacetime is axisymmetric.

There appears to be a small trend towards spin-angular momentum alignment. To observe this effect we measure the angle between the spin and final angular momentum (i.e. the remnant spin), $\theta_{\mathrm{JS}}=\arccos \left(\hat{S} \cdot \hat{J}_{\mathrm{f}}\right)$. In Fig. 17 we show $\theta_{\mathrm{JS}}$ versus time for the SP3 and SP4 configurations using both $\vec{S}_{\mathrm{IH}}$ and $\vec{S}_{\text {coord }}$. Both of these measurements show increasingly larger oscillations in $\theta_{\mathrm{JS}}$ versus time. The $\vec{S}_{\mathrm{IH}}$ based measurements indicate that the spins in the SP3 configuration migrate $\sim 6^{\circ}$ towards $\hat{J}_{\mathrm{f}}$, while the spins in the SP4 configuration migrate $\sim 8^{\circ}$ towards $\hat{J}_{\mathrm{f}}$ (we measure the angle at the approximate midpoint of the oscillation). However, the $\vec{S}_{\text {coord }}$ based measurements indicate that the spins in the SP3 configuration migrate by only $\sim 3^{\circ}$, while the spins in the SP4 configuration migrate by only $\sim 2.5^{\circ}$. It is unclear which measurement is superior. On the one hand $\vec{S}_{\text {coord }}$ is more strongly coordinate dependent, but, on the other hand, the horizons become more distorted as they approach each other, which increases the error in $\vec{S}_{\mathrm{IH}}$ (i.e. the horizons deviate increasingly strongly from axisymmetry). This is an interesting effect which we plan to study in much more detail with improved techniques to measure the spin direction. We summarize the main results from spin direction calculations for the SP3 and SP4 simulation in Table II.

TABLE II. The angle between the initial spin direction and orbital angular momentum $\vartheta$, the total spin precession angle $\Theta_{p}$, spin flip angle between the initial spin direction and remnant spin direction $\Theta_{\text {flip }}$, and the net change in the $z$-component of the angular momentum as calculated using the isolated horizon spin direction of the remnant $\delta J_{z}(\mathrm{IH})$ and waveform $\delta J_{z}\left(\psi_{4}\right)$ for the SP3 and SP4 configurations.

\begin{tabular}{lccccc}
\hline \hline Config & $\vartheta$ & $\Theta_{p}$ & $\Theta_{\text {flip }}$ & $\delta J_{z}(\mathrm{IH})$ & $\delta J_{z}\left(\psi_{4}\right)$ \\
\hline SP3 & $90^{\circ}$ & $98^{\circ} \pm 2^{\circ}$ & $72^{\circ} \pm 2^{\circ}$ & $0.237 \pm 0.003$ & $0.24 \pm 0.02$ \\
SP4 & $45^{\circ}$ & $151^{\circ} \pm 2^{\circ}$ & $35^{\circ} \pm 2^{\circ}$ & - & - \\
\hline \hline
\end{tabular}

TABLE III. Merger times for the SP3 and SP4 configuration versus resolution, as well as an extrapolation to infinite resolution (see text for an explanation of the "extrapolation" of the SP4 result.)

\begin{tabular}{lcc}
\hline \hline Resolution & SP3 & SP4 \\
\hline$M / 22.5$ & $152.0 \pm 0.2$ & - \\
$M / 25$ & $157.4 \pm 0.2$ & $195.4 \pm 0.2$ \\
$M / 30$ & $164.2 \pm 0.2$ & - \\
$M / \infty$ & $176 \pm 3$ & $\sim 214$ \\
\hline \hline
\end{tabular}


The stability of the spinning binaries is strongly dependent on the direction of the spin. In Table III we show the merger times $\left(T_{\mathrm{CAH}}\right)$ of the SP3 configuration versus resolution and an extrapolation to infinite resolution, as well as the merger time for the single SP4 run. The "extrapolated" value of the SP4 merger time was computed by adding the difference between the extrapolated and $h=M / 25$ merger times for the SP3 configuration to the $h=M / 25$ merger time for the SP4 configuration. The extrapolated values of $176 \pm 3$ and $\sim 214$, for SP3 and SP4, respectively, are in large part consistent with the results from the aligned-spin binaries if we replace $\mathcal{S}$ in Eq. (A1) with $2 S_{z} / m^{2}$ ( $\mathcal{S}$ is the total spin, hence the factor of 2 ). The predicted merger times are $T_{\mathrm{CAH}}=172 \pm 1$ and $T_{\mathrm{CAH}}=200 \pm 2$ for the SP3 and SP4 configurations, respectively. The differences between these predictions and the actual extrapolated merger times can be explained by the net rotation of the component spins towards the $z$-axis, which helps stabilize the binaries.

We conclude this section by showing that the constraint violations converge to third-order. Although the code uses purely fourth-order stencils, lower order errors both from the lower differentiability of the evolved fields at the punctures, as well as from the second-order accuracy of the initial data, lead to a global third-order error in the constraint violation. Figures 18-20 show the Hamiltonian constraint, momentum constraint, and BSSN constraint $\left(G^{i}=\tilde{\Gamma}^{i}+\partial_{j} \tilde{g}^{i j}\right)$ violations at $t=76 M$ (the time when the punctures cross the $x$-axis for the second time) along the $x$-axis for the SP3 configuration. The constraint viola-

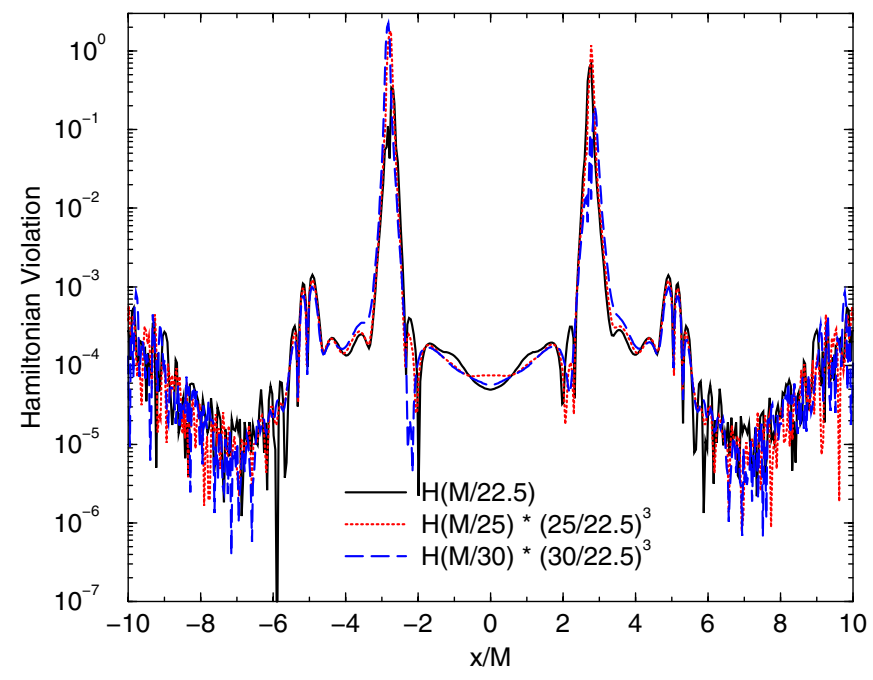

FIG. 18 (color online). The convergence of the Hamiltonian constraint violation for the SP3 configuration along the $x$-axis at $t=76 M$ when the punctures cross the $x$-axis for the second time. The Hamiltonian constraint shows third-order convergence. Points inside the domain of dependence of the boundary have been excluded. The high-frequency features near the outer boundary are due to the extreme fisheye deresolution and converge with resolution.
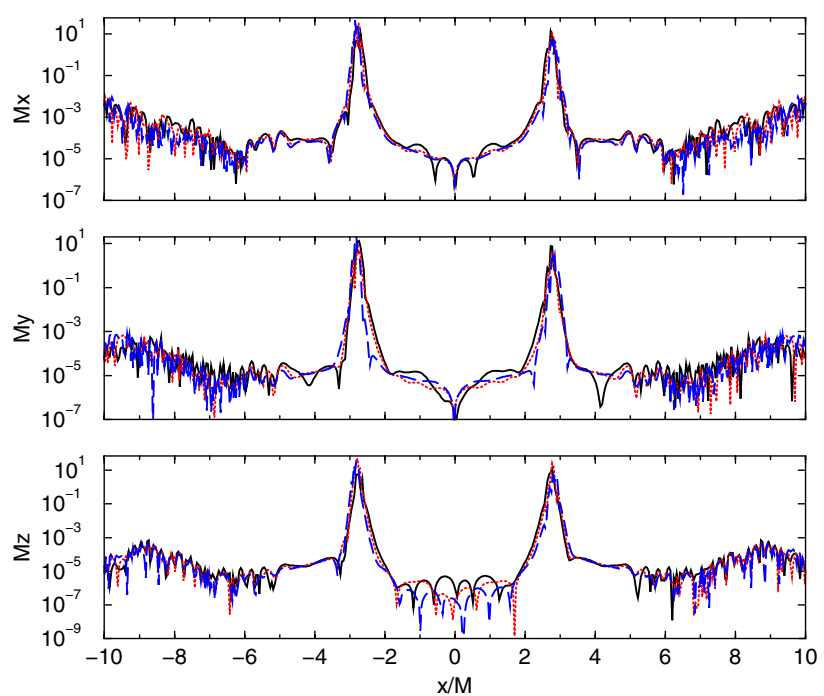

FIG. 19 (color online). The convergence of the momentum constraint violation for the SP3 configuration along the $x$-axis at $t=76 M$ when the punctures cross the $x$-axis for the second time. The momentum constraint shows third-order convergence. Points inside the domain of dependence of the boundary have been excluded. In each panel the solid (black) curve, dotted (red) curve and dashed (blue) curves are the $M / 22.5, M / 25$, and $M / 30$ constraint violations, respectively. The constraints have been rescaled by $\left(h_{l} / h\right)^{3}\left(h_{l}=M / 22.5\right)$. The high-frequency features near the outer boundary are due to the extreme fisheye deresolution and converge with resolution.
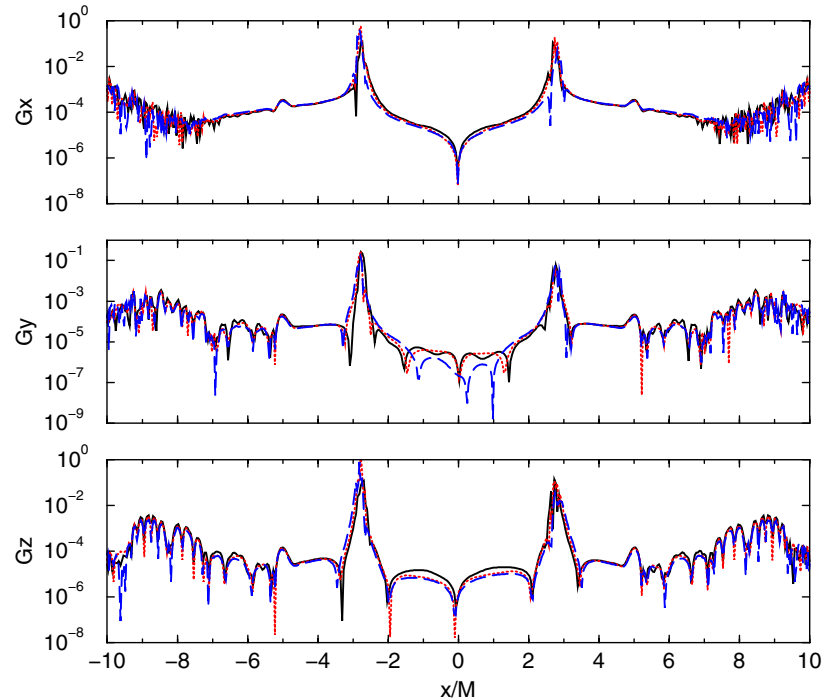

FIG. 20 (color online). The convergence of the BSSN constraint violation for the SP3 configuration along the $x$-axis at $t=$ $76 M$ when the punctures cross the $x$-axis for the second time. The BSSN constraint shows third-order convergence. Points inside the domain of dependence of the boundary have been excluded. In each panel the solid (black) curve, dotted (red) curve and dashed (blue) curves are the $M / 22.5, M / 25$, and $M / 30$ constraint violations, respectively. The constraints have been rescaled by $\left(h_{l} / h\right)^{3}\left(h_{l}=M / 22.5\right)$. The high-frequency features near the outer boundary are due to the extreme fisheye deresolution and converge with resolution. 
tions have been multiplied by $\left(h_{l} / h\right)^{3}$ (where $h_{l}=$ $M / 22.5$ ) to demonstrate third-order convergence. Note that the high-frequency features near the outer boundary are due to the extreme fisheye deresolution near the outer boundary and converges to zero with resolution.

\section{CONCLUSION}

In this paper, we evolved systems of equal-mass and equal-spin black-hole binaries with initial spins aligned perpendicular to, and $45^{\circ}$ to, the orbital angular momentum. We observed the combined effects of spin and orbital plane precession as predicted by post-Newtonian theory, with dramatic, total precessions of $\sim 98^{\circ}$ and $\sim 151^{\circ}$ in the SP3 and SP4 simulations, respectively. Both configurations resulted in large spin flips between the individual horizon spin directions and the final remnant direction, with the SP3 configuration showing a spin flip of $\sim 72^{\circ}$ and the SP4 configuration (which had spins initially more closely aligned with the orbital angular momentum) resulting in a spin flip of $\sim 34^{\circ}$. We see a possible small trend to spinorbital angular momentum alignment, but no evidence for significant spin-up of the black-hole spins.

Although the configurations studied here are paritysymmetric, this symmetry was only chosen to reduce the memory footprint of the simulations (allowing for higher resolution runs); it does not affect the stability of the "moving punctures" algorithm. Notably, the punctures move out of the $x y$ plane and can get arbitrarily close to the numerical grid points. Despite this symmetry, the SP3 and SP4 configurations display most of the significant spinorbit coupling effects associated with spinning binaries: spin and orbital plane precession, spin flips, and enhanced stability of the semialigned configuration. The only significant spin-orbit coupling effect not shown by these configurations is a spin-orbit induced kick of the remnant hole. In order to see these kicks we would need to evolve configurations without parity symmetry.

Our methods for calculating the spin direction produce reasonable results for our choice of gauge conditions. Future work will concentrate on improving this calculation with alternative choices of the gauge parameters (e.g. $\eta$ in the Gamma-driver shift and various different choices of initial values for the lapse function) and with alternative forms of the Gamma-driver shift condition. Notably, the lack of agreement between the location of the horizon semiminor axis and the spin direction indicates that these coordinates are not yet ideal. In addition, it would be useful to calculate independent measures of the quality of the approximate Killing vector, for example, the norm of the Lie derivative of the 2-metric on the horizon $\mathcal{L}_{\varphi} q_{a b}$. It is interesting to note that the purely coordinate measurement $\vec{S}_{\text {coord }}$ gives reasonable results for the spin direction and amplitude, and since this calculation is both more robust (i.e. the approximate Killing vector may not exist) than the approximate Killing vector calculations and easier to implement, it may prove to be a convenient measurement of the spin for those codes that have not implemented the approximate Killing vector finding algorithm.

From the mathematical side, further investigation is required to make the definition of the spin vector more rigorous and gauge independent. There are some interesting questions deserving further attention. Is it possible to meaningfully compare the spin vector defined at the black hole with the vector defined at spatial infinity? What about comparing the spin vector of the final black hole with that for the individual black holes that we start with? Does it matter that they are all calculated on different dynamical horizons? With regard to the latter question, it is possible that the dynamical horizons for the individual black holes are smoothly connected with the final dynamical horizon through the appearance of marginally trapped surfaces lying between the common outer horizon and the two inner horizons. This scenario is suggested by initial numerical studies [57], but further analytical and numerical work is required to confirm this and to fully understand the dynamics of marginally trapped surfaces.

We also revisited the question of tidal locking leading to corotation in close black hole binaries. Corotation implies both that the spin directions are aligned with the orbital angular momentum and that the horizon frequency (the horizon frequency is the angular speed of locally nonrotating observers as they pass through the horizon, as seen by stationary observers at infinity) is equal to the orbital frequency. We found in a previous study [34] that the spin-up of the holes is too small (by 2 orders of magnitude) to reach corotation. In this paper we observed signs of alignment of the spin with the total angular momentum. The variations in the direction observed are a few degrees during the last two orbits. Further numerical and analytic (higher PN order) studies will be needed to see if this effect is strong enough during the slow inspiral phase to drive the binary toward spin-orbit alignment.

Both the final magnitude, and the final direction, of a black-hole binary's remnant spin are of astrophysical interest: the former determines the efficiency of gravitational accretion, and the latter is reflected in the orientation of the inner accretion disk and (indirectly) in the launching direction of a jet. Our simulations are the first to follow the time dependence of the spin orientations in black hole mergers with initially misaligned spins, and the first to verify the spin-flip phenomenon: the sudden reorientation in spin axis that takes place when the binary's orbital angular momentum is converted into spin in the final stages of the merger [10]. In addition to influencing the gravitational wave forms, the spin evolution would also be reflected in any electromagnetic signature due to gas in orbit around the black holes. Predicting the latter signature is beyond the scope of the present paper but is a fruitful topic for further study. 


\section{ACKNOWLEDGMENTS}

We thank Alessandra Buonanno and Kip Thorne for valuable discussions about spin-flips, and Vicky Kalogera for bringing up the papers related to the data analysis of precessing binaries. We thank Erik Schnetter for technical support and for providing the thorns to implement $\mathrm{Pi}$ symmetry boundary conditions, and Marcus Ansorg for providing the TWOPUNCTURES initial data thorn. We gratefully acknowledge the support of the NASA Center for Gravitational Wave Astronomy at University of Texas at Brownsville (NAG5-13396) and the NSF for financial support from Grant No. PHY-0722315. Computational resources were provided by the Funes cluster at UTB, the Lonestar cluster at TACC, and the Tungsten cluster at NCSA.

\section{APPENDIX: PREVIOUS STUDIES}

In a previous paper we reported the first fully-nonlinear studies of highly-spinning black-hole binaries [35], where we found that the spin can profoundly affect the orbital dynamics of the last premerger stages. In Ref. [35] we studied cases where the spins were aligned or counter aligned with the orbital angular momentum. As a result of the spin-orbit coupling the merger times dramatically changed with respect to the nonspinning case. For example, for initial data corresponding to a quasicircular orbit with period $T \sim 125 M$ and orbital frequency $\omega=$ $0.05 / M$, the nonspinning holes would orbit twice before merging into a single horizon, while the spinning holes aligned with the orbital angular momentum and spinning at a rate $S / m^{2}=0.75$ (where $S$ is the magnitude of the spin angular momentum and $m$ is the mass of the black hole) would orbit 3 times before merger. The antialigned spinning holes with specific spins $S / m^{2}=-0.75$ would only complete one orbit before the common event horizon formed. These results can be summarized by a linear fit to the Richardson extrapolated merger times $t_{\mathrm{CAH}}$ (formation time of the first common apparent horizon) of the most accurate runs with $S / m^{2}=0.0, S / m^{2}=0.1$, and $S / m^{2}=$ -0.757 (see Refs. [34,35])

$$
\frac{t_{\mathrm{CAH}}}{M}=(172 \pm 1)+(40 \pm 2) \mathcal{S},
$$

where $\mathcal{S} \equiv\left(S_{1} / m_{1}^{2}+S_{2} / m_{2}^{2}\right)_{I}$. Note that extrapolating to maximally spinning holes gives a merger time (from orbital $\omega=0.05 / M$ ) of $87 M$ and $255 M$ for antialigned and aligned spins, respectively.

Extrapolation to maximally rotating black holes aligned with the orbital angular momentum leads to remnant black holes having a submaximal specific rotation parameter $S / m^{2}<0.95$ which implies one cannot generate extreme rotating black holes or violate the cosmic censorship hypothesis starting from orbiting black holes (see Fig. 21).

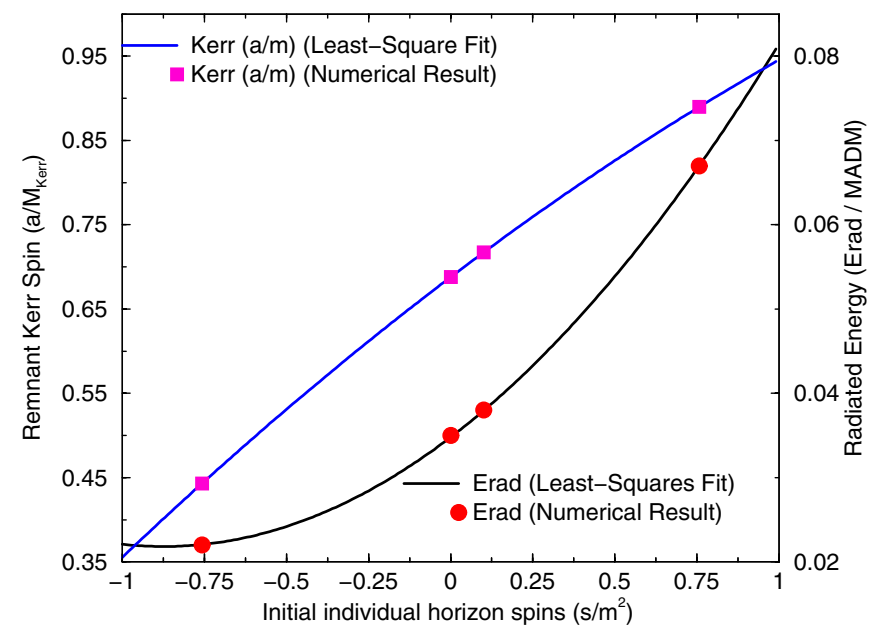

FIG. 21 (color online). A linear-least squares fit of the remnant Kerr spin parameter (left $y$-axis) and radiated energy (right $y$-axis) for the merger of equal-mass equal-spin binaries with spins pointing along (or in the opposite direction to) the orbital angular momentum. The fits have the functional form $y=c_{0}+$ $c_{1}\left(S / m^{2}\right)+c_{2}\left(S / m^{2}\right)^{2}$.

A quadratic fit to the remnant black hole of the merger of aligned or antialigned spinning holes produces

$$
\left.\left(S / M_{H}^{2}\right)\right|_{R}=0.6879+0.1476(\mathcal{S})-0.00935(\mathcal{S})^{2},
$$

while a fit to the energy radiated versus the initial individual spins yields

$$
\frac{E_{\mathrm{rad}}}{M}=0.0348+0.01485(\mathcal{S})+0.00425(\mathcal{S})^{2},
$$

While we expect more simulations of spinning black holes for other values of the individual spins and with even higher accuracy will give improved fits, Eqs. (A1)-(A3) already provide valuable information for data analysts and for theoreticians modeling the merger of spinning blackhole binaries with post-Newtonian or "Kludge" waveforms [63].

This differential orbital dynamics in turn also notably changes waveforms (see Figs. 1-3 in Ref. [35]).

We then explored changes in the magnitude of the spin due to tidal effects in binaries and the transfer of orbital angular momentum to spin and vice versa [34]. Those studies concluded that it is very unlikely that black-hole binaries become tidally locked in a corotating state during the last orbital stages. We considered two representative cases, one starting with initially nonspinning black holes and tracked the spin-up during the last two orbits before merger. The second simulation began with the binary in an instantaneously corotating state at the same starting point and again tracked the spin-up of the individual holes. In both cases the spin-up was 2 orders of magnitude smaller than that needed to lock the binary into a corotating state. 
[1] J.H. Krolik, in Active Galactic Nuclei: From the Central Black Hole to the Galactic Environment, edited by Julian H. Krolik (Princeton University Press, Princeton, NJ, 1999).

[2] M. Elvis, G. Risaliti, and G. Zamorani, Astrophys. J. Lett. 565, L75 (2002).

[3] M. C. Begelman, R. D. Blandford, and M. J. Rees, Rev. Mod. Phys. 56, 255 (1984).

[4] S. Komossa, Mem. Soc. Astron. Ital. 77, 733 (2006).

[5] N. Roos, J. S. Kaastra, and C. A. Hummel, Astrophys. J. 409, 130 (1993).

[6] J. I. Katz, Astrophys. J. 478, 527 (1997).

[7] P. Parma, R. D. Ekers, and R. Fanti, Astron. Astrophys. Suppl. Ser. 59, 511 (1985).

[8] J. P. Leahy and P. Parma, in Extragalactic Radio Sources. From Beams to Jets, edited by J. Roland, H. Sol, and G. Pelletier (Cambridge Univ. Press, Cambridge, England, 1992), p. 307.

[9] J. F. Gallimore, D. J. Axon, C. P. O'Dea, S. A. Baum, and A. Pedlar, Astron. J. 132, 546 (2006).

[10] D. Merritt and R. D. Ekers, Science 297, 1310 (2002).

[11] C. Zier and P. L. Biermann, Astron. Astrophys. 396, 91 (2002).

[12] A. L. Kinney, H. R. Schmitt, C. J. Clarke, J. E. Pringle, J. S. Ulvestad, and R. R. J. Antonucci, Astrophys. J. 537, 152 (2000).

[13] D. Merritt, Astrophys. J. 568, 998 (2002).

[14] D. Merritt and M. Milosavljević, Living Rev. Relativity 8, 8 (2005).

[15] R. Vogt, in Proceedings of the Sixth Marcel Grossman Meeting on General Relativity Kyoto, Japan, 1991, edited by H. Sato and T. Nakamura (World Scientific, Singapore, 1992), pp. 244-266.

[16] F. Acernese et al., Classical Quantum Gravity 23, S635 (2006).

[17] GEO, gEO600 - http://www.geo600.uni-hannover.de/.

[18] C. Cutler and K. S. Thorne, gr-qc/0204090.

[19] K. Danzmann et al., Max Planck Institut für Quantenoptik, Report No. MPQ 177, 1993.

[20] K. Danzmann and A. Rudiger, Classical Quantum Gravity 20, S1 (2003).

[21] F. Pretorius, Phys. Rev. Lett. 95, 121101 (2005).

[22] M. Campanelli, C. O. Lousto, P. Marronetti, and Y. Zlochower, Phys. Rev. Lett. 96, 111101 (2006).

[23] J. G. Baker, J. Centrella, D.-I. Choi, M. Koppitz, and J. van Meter, Phys. Rev. Lett. 96, 111102 (2006).

[24] T. Nakamura, K. Oohara, and Y. Kojima, Prog. Theor. Phys. Suppl. 90, 1 (1987).

[25] M. Shibata and T. Nakamura, Phys. Rev. D 52, 5428 (1995).

[26] T. W. Baumgarte and S. L. Shapiro, Phys. Rev. D 59, 024007 (1998).

[27] S. Brandt and B. Brügmann, Phys. Rev. Lett. 78, 3606 (1997).

[28] B. Brugmann, Int. J. Mod. Phys. D 8, 85 (1999).

[29] M. Alcubierre, B. Brügmann, P. Diener, M. Koppitz, D. Pollney, E. Seidel, and R. Takahashi, Phys. Rev. D 67, 084023 (2003).

[30] M. Campanelli, C. O. Lousto, and Y. Zlochower, Phys. Rev. D 73, 061501(R) (2006).

[31] F. Pretorius, Classical Quantum Gravity 23, S529 (2006).
[32] J. G. Baker, J. Centrella, D.-I. Choi, M. Koppitz, and J. van Meter, Phys. Rev. D 73, 104002 (2006).

[33] J.G. Baker, J.R. van Meter, S.T. McWilliams, J. Centrella, and B. J. Kelly, gr-qc/0612024.

[34] M. Campanelli, C. O. Lousto, and Y. Zlochower, Phys. Rev. D 74, 084023 (2006).

[35] M. Campanelli, C. O. Lousto, and Y. Zlochower, Phys. Rev. D 74, 041501(R) (2006).

[36] F. Herrmann, D. Shoemaker, and P. Laguna, gr-qc/ 0601026.

[37] J. A. Gonzalez, U. Sperhake, B. Bruegmann, M. Hannam, and S. Husa, gr-qc/0610154.

[38] J. G. Baker et al., astro-ph/0603204.

[39] M. Shibata and K. Uryu, astro-ph/0611522.

[40] F. Loffler, L. Rezzolla, and M. Ansorg, Phys. Rev. D 74, 104018 (2006).

[41] M. A. Scheel et al., Phys. Rev. D 74, 104006 (2006).

[42] S. A. Hayward, Phys. Rev. D 49, 6467 (1994).

[43] A. Ashtekar et al., Phys. Rev. Lett. 85, 3564 (2000).

[44] A. Ashtekar and B. Krishnan, Phys. Rev. Lett. 89, 261101 (2002).

[45] A. Ashtekar and B. Krishnan, Living Rev. Relativity 7, 10 (2004).

[46] E. Gourgoulhon and J. L. Jaramillo, Phys. Rep. 423, 159 (2006).

[47] I. Booth, Can. J. Phys. 83, 1073 (2005).

[48] A. Ashtekar, S. Fairhurst, and B. Krishnan, Phys. Rev. D 62, 104025 (2000).

[49] A. Ashtekar, C. Beetle, and J. Lewandowski, Phys. Rev. D 64, 044016 (2001).

[50] I.S. Booth, Classical Quantum Gravity 18, 4239 (2001).

[51] I. Booth and S. Fairhurst, Classical Quantum Gravity 22, 4515 (2005).

[52] A. Ashtekar and B. Krishnan, Phys. Rev. D 68, 104030 (2003).

[53] S. A. Hayward, Phys. Rev. Lett. 93, 251101 (2004).

[54] S. A. Hayward, Phys. Rev. D 74, 104013 (2006).

[55] E. Gourgoulhon and J.L. Jaramillo, Phys. Rev. D 74, 087502 (2006).

[56] O. Dreyer, B. Krishnan, D. Shoemaker, and E. Schnetter, Phys. Rev. D 67, 024018 (2003).

[57] E. Schnetter, B. Krishnan, and F. Beyer, Phys. Rev. D 74, 024028 (2006).

[58] L. E. Kidder, Phys. Rev. D 52, 821 (1995).

[59] B. M. Barker and R.F. O'Connell, Gen. Relativ. Gravit. 11, 149 (1979).

[60] P. Grandclement, V. Kalogera, and A. Vecchio, Phys. Rev. D 67, 042003 (2003).

[61] P. Grandclement and V. Kalogera, Phys. Rev. D 67, 082002 (2003).

[62] P. Grandclement, M. Ihm, V. Kalogera, and K. Belczynski, Phys. Rev. D 69, 102002 (2004).

[63] A. Buonanno, Y. Chen, and T. Damour, Phys. Rev. D 74, 104005 (2006).

[64] A. Buonanno, Y.-b. Chen, and M. Vallisneri, Phys. Rev. D 67, 104025 (2003).

[65] Y. Pan, A. Buonanno, Y.-b. Chen, and M. Vallisneri, Phys. Rev. D 69, 104017 (2004).

[66] A. Buonanno, Y.-b. Chen, Y. Pan, and M. Vallisneri, Phys. Rev. D 70, 104003 (2004). 
[67] M. D. Hartl and A. Buonanno, Phys. Rev. D 71, 024027 (2005).

[68] A. Buonanno, Y. Chen, Y. Pan, H. Tagoshi, and M. Vallisneri, Phys. Rev. D 72, 084027 (2005).

[69] A. Vecchio, Phys. Rev. D 70, 042001 (2004).

[70] M. Ansorg, B. Brügmann, and W. Tichy, Phys. Rev. D 70, 064011 (2004).

[71] cactus_web, cactus Computational Toolkit home p.: http:// www.cactuscode.org.
[72] Y. Zlochower, J. G. Baker, M. Campanelli, and C. O. Lousto, Phys. Rev. D 72, 024021 (2005).

[73] U. Sperhake, gr-qc/0606079.

[74] M. Hannam, S. Husa, D. Pollney, B. Brugmann, and N. O’Murchadha, gr-qc/0606099.

[75] B. Bruegmann et al., gr-qc/0610128.

[76] C. Gundlach and J. M. Martin-Garcia, Phys. Rev. D 74, 024016 (2006). 